\title{
A homogenization method for ductile-brittle composite laminates at large deformations
}

\section{Poulios, Konstantinos; Niordson, Christian Frithiof}

Published in:

International Journal for Numerical Methods in Engineering

Link to article, DOI:

10.1002/nme.5637

Publication date:

2018

Document Version

Peer reviewed version

Link back to DTU Orbit

Citation (APA):

Poulios, K., \& Niordson, C. F. (2018). A homogenization method for ductile-brittle composite laminates at large deformations. International Journal for Numerical Methods in Engineering, 113(5), 814-833 . https://doi.org/10.1002/nme.5637

\section{General rights}

Copyright and moral rights for the publications made accessible in the public portal are retained by the authors and/or other copyright owners and it is a condition of accessing publications that users recognise and abide by the legal requirements associated with these rights.

- Users may download and print one copy of any publication from the public portal for the purpose of private study or research.

- You may not further distribute the material or use it for any profit-making activity or commercial gain

- You may freely distribute the URL identifying the publication in the public portal 


\title{
A homogenization method for ductile-brittle composite laminates at large deformations
}

\author{
Konstantinos Poulios* and Christian F. Niordson \\ Department of Mechanical Engineering, Technical University of Denmark, \\ Nils Koppels Allé, Building 404, 2800 Kgs. Lyngby, Denmark
}

SUMMARY

This paper presents a high fidelity homogenization method for periodically layered composite structures that accounts for plasticity in the matrix material and quasi-brittle damage in the reinforcing layers, combined with strong geometrical nonlinearities. A set of deliberately chosen internal kinematic variables results in a rigorous representation of the kinematics of the two constituents, which in turn allows for complex constitutive laws per constituent to be employed directly in the formulation. The model accounts for hyperelastoplastic behavior in the matrix phase and hyper-elastic behavior in the reinforcement as well as for the bending stiffness of the reinforcement layers. Additionally to previously proposed models, the present method includes Lemaitre type damage for the reinforcement, making it applicable to a wider range of engineering applications. The capability of the proposed method in representing the combined effect of plasticity, damage and buckling at microlevel within a homogenized setting is demonstrated by means of direct comparisons to a reference discrete model. Copyright (c) 2017 John Wiley \& Sons, Ltd.

Received .

KEY WORDS: composite materials; damage; plasticity; large deformations; homogenization

\footnotetext{
*Correspondence to: E-mail: kopo@ mek.dtu.dk

This article has been accepted for publication and undergone full peer review but has not been through the copyediting, typesetting, pagination and proofreading process, which may lead to differences between this version and the Version of Record. Please cite this article as doi: $10.1002 / \mathrm{nme} .5637$
} 


\section{INTRODUCTION}

The importance of composite materials for a plethora of engineering applications motivates the development of accurate and efficient numerical methods for the structural analysis of corresponding components. Among the different types of composites available, the present work focuses on materials with a microstructure of periodically alternating layers of two distinct phases. Although the presented methodology is applicable to a broad range of constitutive behaviors for the two phases, in the present work it is specialized to the combination of a ductile and a quasi-brittle phase. The layered microstructure considered here can also be seen as a two dimensional analog of long fiber reinforced composites. Hence, the term "fiber" will by convention be assigned to the quasibrittle phase, which is typically stiffer and stronger than the ductile phase that will accordingly be denoted as the "matrix". Failure of the quasi-brittle layers can be referred to as fiber breakage or fiber crushing, depending on whether the corresponding layers break under tension or bending or under compression, respectively [1].

Mechanical systems with a microstructure of the aforementioned characteristics can be found e.g. in connection with metallic-ceramic composites [2], co-extruded polymer-polymer laminates [3] or equivalent additively manufactured laminates. Applications of an elastomer matrix instead of a ductile phase are also covered by the present work. Moreover, unidirectional fiber reinforced prepregs could in principle be considered as the quasi-brittle phase as well, but this would require an extension of the material model for the quasi-brittle layers to orthotropic elasticity with damage. Finally, the laminated microstructure considered here, seen as a plane strain approximation of unidirectional fiber reinforced composites in general, is an adequate approximation for studying several effects of fiber breakage in such composites qualitatively.

So-called direct simulation could be applied for studying the micromechanical behavior of the considered laminates by discretizing each individual lamina in the microstructure explicitly. In this context, standard finite-element techniques with constitutive laws of arbitrary complexity for each material phase are rather straightforward to apply as e.g. in [4] for studying compressive instabilities

This article is protected by copyright. All rights reserved. 
and in [5] for tensile loading including damage. Nevertheless, the computational cost of direct simulation limits its applicability to rather small and geometrically simple domains. Accounting for a heterogeneous microstructure in the modeling of larger components or structures requires a homogenized representation.

A practical approach is to neglect the complex microscale phenomena and use simple linearized constitutive laws in the analysis phase, followed by a post-processing step, where the obtained stresses and strains are used as input to well established anisotropic failure criteria that represent the microscale [6]. Alternatively, generic constitutive frameworks for anisotropic plasticity and damage, directly defined at the macroscopic level, can be employed in order to account for progressive damage within the component analysis [7, 8]. Such models are often tuned to represent particularly well specific phenomena of interest at the microscale $[9,10]$ and they are also often used in combination with fitting to experimental data.

A third approach, at a higher level of detail, is the representation of heterogeneous microstructures by computational homogenization and so-called multiscale methods, reviewed recently in [11], [12], [13] and [14]. The main problem that homogenization methods deal with in general is how the macroscopic kinematics are transformed into the microstructure kinematics and how the resulting stresses in the microstructure affect the force equilibrium at the macroscale. As an outcome, a macroscopic material response is obtained based on assumed material laws for the different phases contained in the microstructure and the geometrical features of the microstructure. In this process, geometrically complex microstructures, inelastic constituents, damage effects and kinematic nonlinearities present a significant challenge.

Depending on whether the response of the microstructure is determined beforehand or as part of the macroscopic simulation, multiscale methods are divided into two categories. Methods characterized in the literature as sequential, serial or hierarchical reformulate the response of the microstructure into some effective macroscopic behavior that can be used later in a macroscale simulation. Normally, the format of this transfer from the micro- to the macroscale is less abstract than in the case of the aforementioned generalized constitutive frameworks but some overlapping 
between the two categories exists. On the other hand, multiscale methods referred to as concurrent or integrated ones solve the microscale problem as part of the macroscale one, normally in a nested manner. An exception to the aforementioned distinction between sequential and concurrent methods is related to modern reduced order modeling techniques. Although the microstructure analysis is performed offline, these methods can potentially provide sufficiently accurate representations of the microstructural response for them to classify as concurrent methods $[15,16]$.

Homogenization techniques and corresponding multiscale methods can also be categorized according to the kinematic variables used to describe the homogenized continuum at the macroscale. The vast majority of homogenization methods in the literature refer to a macroscale continuum that is kinematically described by the displacements field exclusively, with first order homogenization employing only first derivatives of this field, while second order homogenization additionally accounts for second derivatives. It should be stressed here that the restriction of the macroscale kinematic description to the displacements only limits the interface between the two scales to a rather specific format which in turn affects the complexity of the microscale boundary value problem to solve. The discussions found in the literature with respect to alternative boundary conditions for the microscale problem $[17,18]$ are strongly related to the choice of the displacements field as the unique kinematic field at the macroscale. As an alternative, homogenization methods can be applied with respect to a generalized continuum description at the macroscale that includes additional internal kinematic variables [19]. This approach has recently gained popularity resulting in rather successful representations of the underlying microstructure in cases involving relatively complex microstructures [20] as well as damage and plasticity in masonry structures [21, 22]. Also the model proposed in [23] actually homogenizes a ductile-elastic periodic laminate into a macroscopic Cosserat medium, accounting for finite rotations and the bending stiffness of the reinforcing elastic layers. Instead of Cosserat microrotations, the model proposed by the authors [24] employs a non-standard internal kinematic variable in combination with hyper-elastoplasticity at the constituent level in order to additionally provide a valid formulation for large deformations both at the macroscale and within the microstructure.

This article is protected by copyright. All rights reserved. 


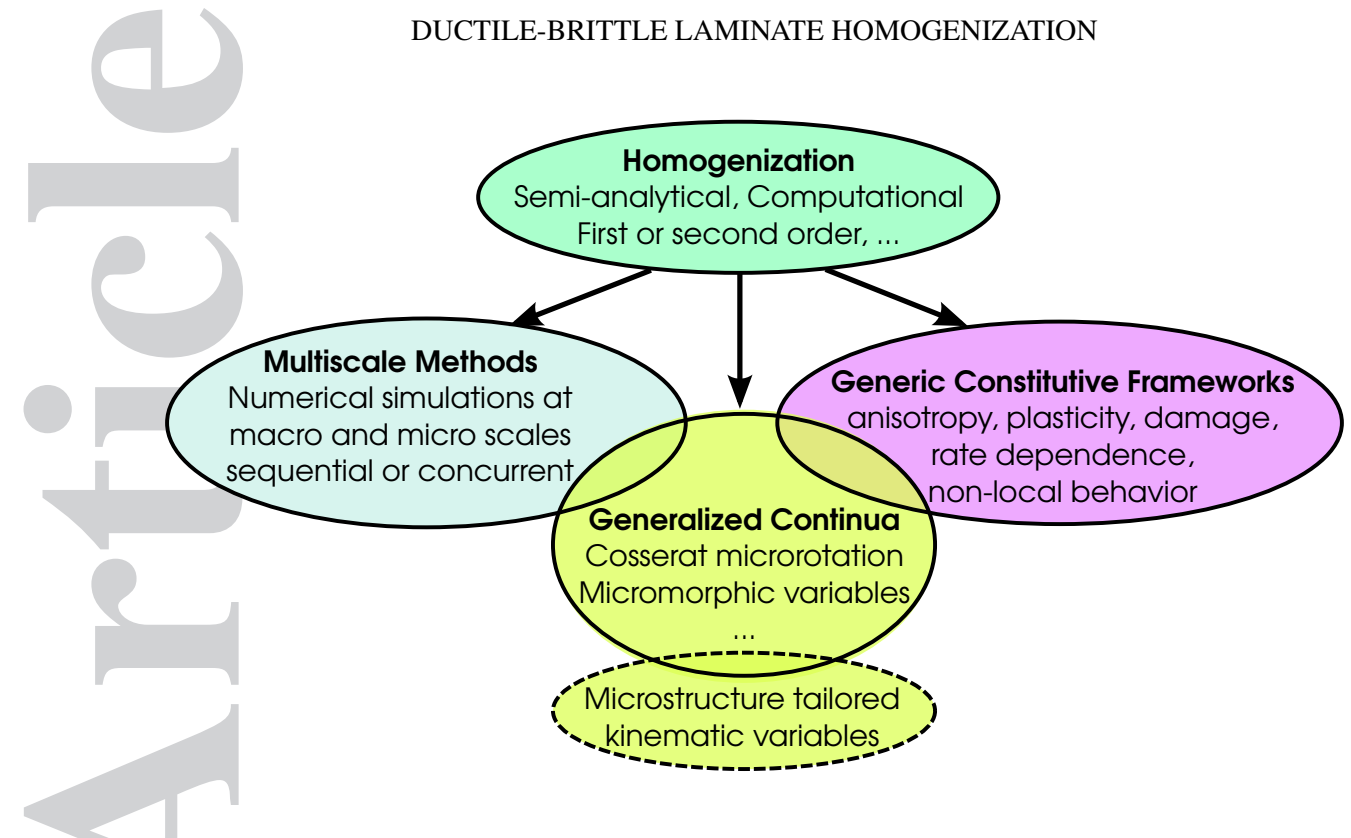

Figure 1. Simplified overview of applications of homogenization in solid mechanics.

Figure 1 shows a simplified overview of the aforementioned categories of solid mechanics models related to homogenization. The notion of generalized continua is extended here to include internal kinematic variables that are tailored to the kinematics of a specific microstructure. The authors' previous work [24] has shown that an appropriate choice of such internal kinematic variables can result in an accurate representation of the considered microstructure, including nonlinear kinematics, without the need for solving a microscale equilibrium problem at each quadrature point.

This elimination of the nested solution required otherwise is at the cost of the additional degrees of freedom due to the kinematic variables at the macroscale. By solving for these variables and the homogenized displacements simultaneously, the microscale and macroscale equilibria are both solved at the macroscale.

Especially regarding the treatment of complex inelastic and load path dependent behavior at the constituent level, multiscale models can be ranked according to the level of abstraction that each framework superposes to the underlying material laws of the individual constituents. In relation to damage, one of the early efforts to relate directly to the constitutive laws of the constituents is the mixing rule proposed in [25]. Later, mathematical homogenization was used in [26] for deriving a continuum damage mechanics model that links directly to damage models of individual elastic constituents, including corresponding damage state variables which are assumed as constant This article is protected by copyright. All rights reserved. 
per constituent. The model introduced in [27] based on the transformation field analysis method additionally accounts for inelastic constituents. Moreover, high fidelity methods such as $\mathrm{FE}^{2}$ [12] and the high fidelity generalized method of cells [28] are rather versatile with respect to the employed constitutive laws within the considered microscale unit cell.

Despite the great progress in the transfer of complex material behaviors from the microscale to the macroscale, indicated by the cited references, the combination of plasticity and damage at the microscale with large deformations both at the macro and micro scales, is still challenging to achieve in a computationally efficient, generic and possibly simple manner. The present work showcases that a generalized kinematic description at the macroscale can lead to a high fidelity representation of a microstructure with rather complex material response per constituent and effects of nonlinear kinematics. A direct link to the quasi-brittle and hyper-elastoplastic constitutive laws respectively for the reinforcing layers and the matrix is maintained in the final formulation. Although averaged stress like quantities at the macroscale, work conjugate to the kinematic variables, are made available in closed form, they are not considered as an essential component of the proposed formulation but rather a technicality.

The rest of this paper is organized in five subsequent sections. Section 2 presents a kinematic description of the considered unit cell along with its embedding in the homogenized setting. Section 3 describes the postulated constitutive laws for the two constituents and finally derives a virtual work expression with respect to the kinematic variables defined in the previous section. Section 4 presents the necessary discretization steps at unit cell and global level along with an algorithm for tracking the solution through global instabilities in a quasi-static approximation. Section 5 contains numerical results including comparisons with a reference discrete model and Section 6 summarizes and concludes the paper.

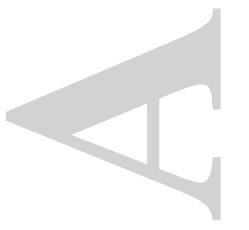

This article is protected by copyright. All rights reserved. 


\section{KINEMATICS}

In this work, a periodically layered microstructure is considered, consisting of two types of alternating layers subjected to plane strain conditions. The volumetric ratio of the two phases as well as the microstructure orientation angle in the undeformed configuration are considered as given and can vary smoothly across the modeled domain, i.e. the considered laminate is in general a quasiperiodic one. The kinematics of both phases can in principle be treated at the same level of detail. However, here it is anticipated that the comparatively stiffer but quasi-brittle fiber layers require a richer kinematic description than the matrix layers because of possible strong variations of damage through the layer thickness.

One way of looking at the kinematics of the considered composite is by defining two sets of lines $L_{T}$ and $L_{N}$ in the undeformed configuration, respectively tangential and normal to the layers of the microstructure, as depicted in Figure 2. Upon deformation, line segments within $L_{T}$ change orientation, length and curvature, leading to the deformed set of lines $\hat{l}_{T}$ which remain smooth to the degree that the underlying layers do so. On the contrary, segments of the set $L_{N}$, initially oriented normal to the layers of the microstructure, turn into the set of jagged lines $\hat{l}_{N}$ in the deformed configuration with a wavelength corresponding to the period of the considered microstructure. A homogenized interpretation of the underlying inhomogeneous material can be obtained by defining the displacements field $u$ that transforms the two line sets $L_{T}$ and $L_{N}$ into the smoothened line sets $l_{T}$ and $l_{N}$, where $l_{T}$ coincides with $\hat{l}_{T}$ and $l_{N}$ is obtained from $\hat{l}_{N}$ by averaging out the aforementioned micro-fluctuations.

Any arbitrary point $X$ of the reference configuration translates in the deformed configuration into a point $\hat{x}=X+\hat{u}$ through the non-homogenized displacements field $\hat{u}$ and at the same time it can be mapped to a point $x=X+u$ based on the homogenized displacements field $u$. The corresponding deformation gradients are $\hat{F}=I+\nabla \hat{u}$ and $F_{u}=I+\nabla u$, respectively. By the aforementioned definition of the homogenized continuum, a tangent vector to a line in $l_{N}$ has to be the average of the tangent to the corresponding jagged line in $\hat{l}_{N}$ traversing the two constituents, 


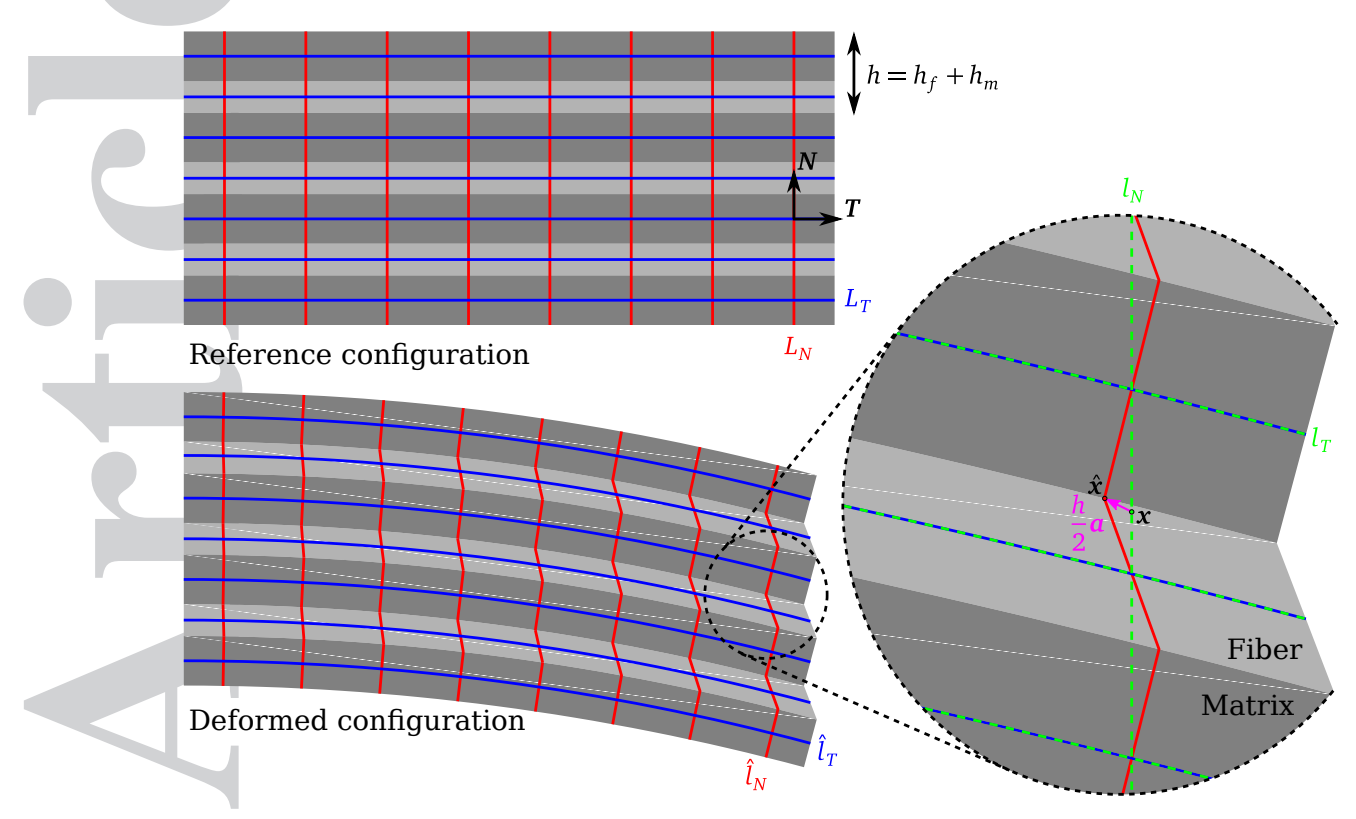

Figure 2. Transformation of reference lines $L_{T}$ and $L_{N}$ to the deformed ones $\hat{l}_{T}$ and $\hat{l}_{N}$ and definition of the internal kinematic variable $a$.

both in terms of direction and length. With $N$ denoting the normalized direction vector of the corresponding undeformed line in $L_{N}$, this last condition translates to

$$
F_{u} N=\frac{1}{h_{f}+h_{m}} \int_{-\left(h_{f}+h_{m}\right) / 2}^{\left(h_{f}+h_{m}\right) / 2} \hat{F}(\chi) N d \chi,
$$

where $\chi$ is a coordinate with origin at an arbitrary point $X$ that describes the position along the line of the set $L_{N}$ passing through $X$.

At the same time, an equivalent expression with respect to the set of tangent lines $l_{T}$ has to be fulfilled both for the fiber and matrix independently:

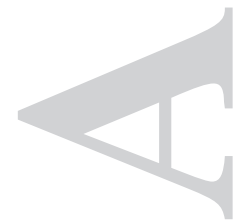

$$
F_{u} T=\frac{1}{h_{f}} \int_{-h_{f} / 2}^{h_{f} / 2} \hat{F}\left(\chi_{f}\right) T d \chi_{f}=\frac{1}{h_{m}} \int_{-h_{m} / 2}^{h_{m} / 2} \hat{F}\left(\chi_{m}\right) T d \chi_{m},
$$

where $T$ is the direction vector linked to $L_{T}, \chi_{f}$ and $\chi_{m}$ are signed distances along the coordinate $\chi$ originating at the fiber and matrix centerlines respectively. The equality of the last two integrals in This article is protected by copyright. All rights reserved. 
Eq. (2) is simply the Voigt condition between the fiber and the matrix phases along the direction of the reinforcement.

Obviously, $F_{u}$ cannot represent the current deformation state of the individual constituents, unless it is used as a boundary condition in a unit cell boundary value problem that has to be solved, based on information about the constitutive behavior of the two phases. As an alternative to this nested approach, a richer kinematic description of the homogenized continuum can be sought, in the form of a generalized continuum with internal kinematic variables. In the authors' previous work, [24], the non-homogenized deformation tensor $\hat{F}$ was approximated by

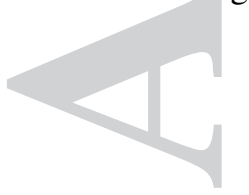

$$
F_{m}=I+\nabla u+\frac{1}{c_{m}} a N^{T}
$$

in the matrix phase and by

$$
\tilde{F}_{f}\left(\chi_{f}\right)=I+\nabla u-\frac{1}{c_{f}} a N^{T}-\chi_{f} \frac{1}{c_{f}} \nabla a T T^{T}
$$

in the fiber phase, where $c_{m}$ and $c_{f}$ are the corresponding volume fractions and $a$ is a two dimensional internal kinematic variable defined in Figure 2. Considering $X$ as a point on the interface between the fiber and the matrix layers at $\chi_{f}=h_{f} / 2$, the strain like internal kinematic variable $a$ is defined as the vector connecting the corresponding points $x$ and $\hat{x}$ in the deformed configuration divided by the half period length $\left(h_{f}+h_{m}\right) / 2$.

The deformation tensors $F_{m}$ and $\tilde{F}_{f}$ defined in Eqs. (3) and (4) were constructed to fulfill Eqs. (1) and (2) by definition. In order to capture the fiber bending kinematics, the tensor $\tilde{F}_{f}\left(\chi_{f}\right)$ was additionally designed to fulfill the following compatibility equation

$$
\frac{\partial \tilde{F}_{12}}{\partial X_{1}}-\frac{\partial \tilde{F}_{11}}{\partial X_{2}}=0 \quad \text { and } \quad \frac{\partial \tilde{F}_{22}}{\partial X_{1}}-\frac{\partial \tilde{F}_{21}}{\partial X_{2}}=0
$$

which ensures that there exists an underlying continuum that the tensor $\tilde{F}$ is the deformation gradient of. It should be noted that gradients of the field $a$ along direction $N$, i.e. perpendicular to the 
composite layers, must be excluded from the evaluation of the compatibility Eq. (5) because they express interlayer variations rather than variations within a single reinforcing layer. The latter are captured exclusively through the dependence on the local coordinate $\chi_{f}$.

The finite-element method presented in the sequel is based on the insight that the deformation gradient $\tilde{F}_{f}\left(\chi_{f}\right)$ enhanced with the internal kinematic variable $a$ and its spatial gradient $\nabla a$ is just the simplest of an infinite number of tensor spaces in the interval $-h_{f} / 2 \leq \chi_{f} \leq h_{f} / 2$ that fulfill Eqs. (1), (2) and (5). In fact, any tensor defined as

$\tilde{F}_{f}\left(\chi_{f}\right)=I+\nabla u-\frac{1}{c_{f}}\left(a+\sum_{i} w_{i}\left(\chi_{f}\right) b_{i}\right) N^{T}-\frac{1}{c_{f}}\left(\chi_{f} \nabla a+\sum_{i} g_{i}\left(\chi_{f}\right) \nabla b_{i}\right) T T^{T}$

fulfills the aforementioned conditions for any set of smooth vector fields $b_{i}$, provided that:

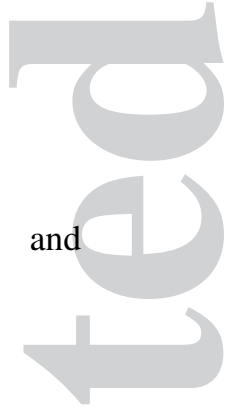

$$
\int_{-h_{f} / 2}^{h_{f} / 2} w_{i}\left(\chi_{f}\right) d \chi_{f}=\int_{-h_{f} / 2}^{h_{f} / 2} g_{i}\left(\chi_{f}\right) d \chi_{f}=0 \quad \forall i
$$

Some possible choices for enrichment functions $w_{i}$ and $g_{i}$ are presented in subsection 4.1. Once a choice has been made for the exact form of Eq. (6), stress like quantities work conjugate to the kinematic quantities $\nabla u, a, \nabla a, b_{i}$ and $\nabla b_{i}$ can be determined.

\section{CONSTITUTIVE MODELS AND WEAK FORMULATION}

In this section, virtual work expressions are derived with respect to variations $\delta u, \delta a$ and $\delta b_{i}$, which involve a hyper-elastoplastic constitutive law with arbitrary strain hardening for the matrix phase and a hyper-elastic constitutive law with Lemaitre type damage for the fiber phase. In order to be able to employ independent constitutive laws per constituent, the closed form expressions for the deformation tensors in the matrix and fiber phases in terms of the homogenized displacements $u$ and 
internal kinematic variables $a$ and $b_{i}$, provided in Eqs. (3) and (6), are essential. The comparatively simpler representation of the matrix layers according to Eq. (3) is a deliberate choice made here in order to keep the number of internal kinematic variables low. Nevertheless, the richer kinematic description used for the fiber layers could be applied in an equivalent manner to the matrix layers as well.

For both phases, the assumed constitutive models are based on the (non exponentiated) Hencky hyper-elastic material model according to [29] that results in the relationship

$$
\tau=K \ln |F| I+G \operatorname{dev}\left(\ln \left(F F^{T}\right)\right),
$$

between the elastic deformation tensor $F$ and the corresponding Kirchhoff stress tensor $\tau$. The bulk and shear moduli $K$ and $G$ respectively, are substituted with the corresponding quantities $K_{m}$ and $G_{m}$ for the matrix and $K_{f}$ and $G_{f}$ for the fiber phase.

\subsection{Isotropic plasticity}

Assuming associated $\mathrm{J}_{2}$-flow plasticity for the matrix material, the Kirchhoff stress tensor $\tau_{m}$ within the matrix phase is given by Eq. (9) after substituting the deformation tensor $F$ inside the deviatoric part of the equation with the elastic part $F_{m}^{e}$ of the deformation tensor $F_{m}$ in the matrix, resulting in

$$
\tau_{m}=K_{m} \ln \left|F_{m}\right| I+G_{m} \operatorname{dev}\left(\ln \left(F_{m}^{e} F_{m}^{e T}\right)\right) .
$$

The elastic and plastic parts of the deformation tensor in the matrix, denoted as $F_{m}^{e}$ and $F_{m}^{p}$ respectively, are defined through the usual multiplicative split

$$
F_{m}=F_{m}^{e} F_{m}^{p}
$$

Assuming that the plastic deformation in the previous time step $t_{*}$ is known in terms of the corresponding inverse right Cauchy-Green strain tensor $C_{m *}^{p}{ }^{-1}=\left(F_{m *}^{p}{ }^{T} F_{m *}^{p}\right)^{-1}$, a trial version of This article is protected by copyright. All rights reserved. 
the term $F_{m}^{e} F_{m}^{e T}$ can be defined based on the previously available $F_{m *}^{p}$ instead of its current value $F_{m}^{p}$. Such a trial quantity, in combination with the fact that the plastic flow direction in $\mathrm{J}_{2}$-flow plasticity is given by the deviatoric part of Eq. (10) and application of backward Euler time integration, leads to a closed-form expression for the logarithmic elastic strain tensor as a function of $F_{m}$ and an unknown plasticity multiplier $\xi_{m}$, defined as

$$
\ln \left(F_{m}^{e} F_{m}^{e T}\right)=\ln \left(F_{m} C_{m *}^{p}{ }^{-1} F_{m}{ }^{T}\right)-\xi_{m} \operatorname{dev}\left(\ln \left(F_{m} C_{m *}^{p}{ }^{-1} F_{m}{ }^{T}\right)\right)
$$

For further details on the derivation of Eq. (12) the reader is referred to [30] and [24].

Isotropic hardening laws rely on a cumulative plastic strain measure. The current cumulative plastic strain in the matrix, denoted by $\gamma_{m}$, can be evaluated based on the corresponding value $\gamma_{m *}$ in the previous time step, the kinematic variables defining $F_{m}$ and the plasticity multiplier $\xi_{m}$ as

$$
\gamma_{m}\left(\nabla u, a, \xi_{m}\right)=\gamma_{m *}+\xi_{m}\left\|\operatorname{dev}\left(\ln \left(F_{m} C_{m *}^{p}{ }^{-1} F_{m}{ }^{T}\right)\right)\right\|,
$$

which implies a backward Euler time discretization with respect to $\gamma_{m}$.

Given a yield surface $f_{m}\left(\tau_{m}, \gamma_{m}\right)=0$ and knowing that $\tau_{m}$ and $\gamma_{m}$ are functions of $\nabla u, a$ and $\xi_{m}$, the plasticity multiplier $\xi_{m}$ can be determined by enforcing the corresponding yield consistency condition

$$
\begin{cases}f_{m}\left(\nabla u, a, \xi_{m}\right)=0 & : \text { if } f_{m}>0 \\ \xi_{m}=0 & : \text { if } f_{m} \leq 0\end{cases}
$$

For isotropic linear hardening for instance, with initial yield stress $\sigma_{y m}$ and hardening modulus $H_{m}$, the yield function $f_{m}$ is defined as

$$
f_{m}\left(\nabla u, a, \xi_{m}\right)=\left\|\operatorname{dev}\left(\tau_{m} /\left|F_{m}\right|\right)\right\|-\sqrt{2 / 3}\left(\sigma_{y m}+\sqrt{2 / 3} H_{m} \gamma_{m}\right),
$$

For monotonic hardening laws as for the linear hardening law of Eq. (15) with $H_{m} \geq 0$, the pair of conditions in Eq. (14) can be rewritten in a more compact form with the help of the nonsmooth 
yield function

$$
\begin{aligned}
\hat{f}_{m}\left(\nabla u, a, \xi_{m}\right)= & \left\|\operatorname{dev}\left(\tau_{m} /\left|F_{m}\right|\right)\right\| \\
& -\min \left\{\sqrt{2 / 3}\left(\sigma_{y m}+\sqrt{2 / 3} H_{m} \gamma_{m}\right),\left\|\operatorname{dev}\left(\left.\tau_{m}\right|_{\xi_{m}=0} /\left|F_{m}\right|\right)\right\|\right\} .
\end{aligned}
$$

Making use of the latter, Eq. (14) can be replaced by the nonlinear and nonsmooth equality

$$
\hat{f}_{m}\left(\nabla u, a, \xi_{m}\right)=0
$$

that can be directly used in the Newton-Raphson solution of each implicit time integration step, given the plastic strain field $\gamma_{m *}$ at the previous time instant.

\subsection{Quasi-brittle damage}

In order to account for quasi-brittle damage in the fiber material, deterioration of the material stiffness has to be incorporated into the hyper-elastic constitutive law of Eq. (9). For this purpose, the strain based continuum damage model suggested in [31] is taken as a point of departure. Similar to the aforementioned work, an explicit deterioration of the material stiffness is postulated as a function of a damage variable $d$ or the corresponding integrity variable $\omega=1-d$. In particular, the Kirchhoff stress tensor at any distance $\chi_{f}$ from the fiber centerline is assumed to be given by the following damage aware extension of Eq. (9) to

$$
\tilde{\tau}_{f}=K_{f}\left(\tilde{\omega}\left\langle\ln \left|\tilde{F}_{f}\right|\right\rangle-\left\langle-\ln \left|\tilde{F}_{f}\right|\right\rangle\right) I+\tilde{\omega} G_{f} \operatorname{dev}\left(\ln \left(\tilde{F}_{f} \tilde{F}_{f}^{T}\right)\right)
$$

where the Macauley brackets notation $\langle\cdot\rangle$ is used for the ramp function and the identity $x=\langle x\rangle-\langle-x\rangle$ is exploited. With the tilde symbol emphasizing dependence on $\chi_{f}$ throughout the paper, the integrity variable $\tilde{\omega}$ refers to the value of $\omega$ at the transverse position $\chi_{f}$. Eq. (18) simply states that for a damaged material point corresponding to integrity $\tilde{\omega}<1$, the bulk modulus $K_{f}$ remains unaffected in the case of volumetric compression but deteriorates linearly with $\tilde{\omega}$ for volumetric expansion. The shear modulus $G_{f}$ deteriorates linearly with $\tilde{\omega}$, unconditionally. 
A second necessary component for defining a damage model is a law that determines the evolution of $\tilde{\omega}$. Strain based damage models express this evolution in terms of some equivalent strain measure. Here, following the same approach as in [31], an equivalent strain is defined as

$$
\tilde{r}=\sqrt{K_{f}\left\langle\ln \left|\tilde{F}_{f}\right|\right\rangle^{2}+\frac{G_{f}}{2}\left\|\operatorname{dev}\left(\ln \left(\tilde{F}_{f} \tilde{F}_{f}^{T}\right)\right)\right\|^{2}} .
$$

The expression under the square root is equal to two times the undamaged hyper-elastic strain energy density corresponding to Eq. (9), except for any compressive volumetric contributions, which are excluded through the Macauley brackets in Eq. (19). Consequently, the adopted strain measure has units corresponding to the square root of energy density. Nevertheless, it should not be interpreted as a measure of the actually stored elastic energy since it always refers to the undamaged solid.

Assuming that no damage occurs below an equivalent strain threshold $r_{\text {th }}$, an explicit relationship between $\tilde{r}$ and a trial value for the damage $\tilde{d}$ can be postulated as

$$
\tilde{d}_{\text {trial }}=d_{\infty}\left(1-e^{-C_{d}\left\langle\tilde{r}-r_{\text {th }}\right\rangle}\right)
$$

where the coefficient $C_{d}$ defines the slope of the damage evolution, with higher values corresponding to lower material toughness. The parameter $d_{\infty}=1-\omega_{\infty}$ corresponds to a residual integrity $\omega_{\infty}$ very close to one, adopted for numerical reasons.

Considering the complete history of a material point until the current time instant $t$, the current value of the damage variable is defined as an extremum of $\tilde{d}_{\text {trial }}$ within the time interval $[0, t]$, given by

$$
\tilde{d}=\sup _{0 \leq \bar{t} \leq t} \tilde{d}_{\text {trial }}(\bar{t})
$$

A discrete version of the latter reads

$$
\tilde{d}=\max \left\{\tilde{d}_{*}, \tilde{d}_{\text {trial }}\right\}
$$


where $d_{*}$ is a damage value stored at the previous discrete time instant $t_{*}$ and all quantities without an explicit time specifier refer to the current time $t$.

Eqs. (19), (20) and (21), along with the definition $\tilde{\omega}=1-\tilde{d}$, transform Eq. (18) into a closed form expression for $\tilde{\tau}_{f}$ as a function of $\tilde{F}_{f}$ and the known quantity $\tilde{d}_{*}$. Looking at a single time step from $t_{*}$ to $t$, Eq. (18) can be simply seen as a nonlinear and nonsmooth elasticity law, obtained thanks to the time discretization of the damage evolution assumed in Eq. (22).

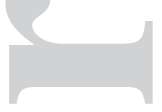

\subsection{Virtual work}

In order to formulate a continuum mechanics model based on the kinematic variables $u, a$ and $b_{i}$ defined earlier, a standard generalized continuum approach would suggest to derive stress like quantities work conjugate to these kinematic variables and seek constitutive laws that express such stresses as a function of the considered kinematic variables. An important realization of this work is that the latter step, namely the derivation of constitutive relationships directly between the kinematic quantities and the corresponding stress like quantities, is not strictly necessary and can actually limit the fidelity in the representation of the underlying micromechanical behavior unnecessarily. Especially for complex constitutive laws in the individual phases that involve state variables e.g. for plasticity and damage, transfer of these quantities to their equivalents expressed in terms of the introduced homogenized stress like quantities can be challenging.

As an alternative, it is possible to work directly with the constitutive laws of the constituents by making an integration over the considered unit cell part of the formulation instead of employing it as an a priori averaging procedure leading to a new constitutive law. In the earlier work by the authors [24], this integration procedure corresponds to a simple weighted averaging of the two phases and an analytical integration through the fiber thickness with respect to fiber bending terms. In the present work, a numerical integration through the fiber thickness is proposed in order to deal with the higher complexity of the assumed constitutive law in the fiber.

One first step in the proposed formulation is the transformation of virtual variations $\delta u, \delta a$ and $\delta b_{i}$ of the so far considered kinematic variables into corresponding variations of the deformation This article is protected by copyright. All rights reserved. 
tensors in the matrix and the fiber phases as

$$
\begin{gathered}
\delta F_{m}=\nabla \delta u+\frac{1}{c_{m}} \delta a N^{T} \\
\text { and } \tilde{F}_{f}\left(\chi_{f}\right)=\nabla \delta u-\frac{1}{c_{f}}\left(\delta a+\sum_{i} w_{i}\left(\chi_{f}\right) \delta b_{i}\right) N^{T}-\frac{1}{c_{f}}\left(\chi_{f} \nabla \delta a+\sum_{i} g_{i}\left(\chi_{f}\right) \nabla \delta b_{i}\right) T T^{T}
\end{gathered}
$$

respectively.

Work conjugate to these variations are the first Piola-Kirchhoff stress tensors in the fiber and matrix phases, respectively defined as

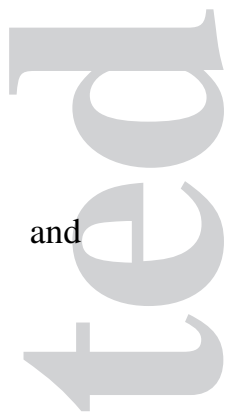

$$
\begin{gathered}
\tilde{P}_{f}=\tilde{\tau}_{f} \tilde{F}_{f}-T \\
P_{m}=\tau_{m} F_{m}{ }^{-T} .
\end{gathered}
$$

Making use of the assumption that neither the stress nor the deformation tensor vary across the matrix layer thickness significantly, the averaged virtual work per undeformed unit volume of the considered microstructure can be written as

$$
\delta W=c_{m} P_{m}: \delta F_{m}+c_{f} \frac{1}{h_{f}} \int_{-h_{f} / 2}^{h_{f} / 2} \tilde{P}_{f}\left(\chi_{f}\right): \delta \tilde{F}_{f}\left(\chi_{f}\right) d \chi_{f}
$$

After substitution of Eqs. (23) and (24) into Eq. (27) the latter can be expanded to

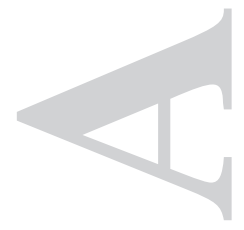

$$
\begin{aligned}
\delta W= & \left(c_{m} P_{m}+c_{f} P_{f 0}\right): \nabla \delta u \\
& +\left(\left(P_{m}-P_{f 0}\right) N\right) \cdot \delta a-\left(P_{f 1} T T^{T}\right): \nabla \delta a \\
& -\sum_{i}\left\{\left(P_{f w(i)} N\right) \cdot \delta b_{i}+\left(P_{f g(i)} T T^{T}\right): \nabla \delta b_{i}\right\},
\end{aligned}
$$

This article is protected by copyright. All rights reserved. 
with

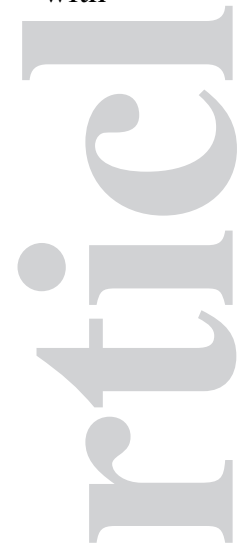

$$
\begin{gathered}
P_{f 0}=\frac{1}{h_{f}} \int_{-h_{f} / 2}^{h_{f} / 2} \tilde{P}_{f}\left(\chi_{f}\right) d \chi_{f}, \\
P_{f 1}=\frac{1}{h_{f}} \int_{-h_{f} / 2}^{h_{f} / 2} \chi_{f} \tilde{P}_{f}\left(\chi_{f}\right) d \chi_{f}, \\
P_{f w(i)}=\frac{1}{h_{f}} \int_{-h_{f} / 2}^{h_{f} / 2} w_{i}\left(\chi_{f}\right) \tilde{P}_{f}\left(\chi_{f}\right) d \chi_{f}, \\
P_{f g(i)}=\frac{1}{h_{f}} \int_{-h_{f} / 2}^{h_{f} / 2} g_{i}\left(\chi_{f}\right) \tilde{P}_{f}\left(\chi_{f}\right) d \chi_{f} .
\end{gathered}
$$

Eq. (28) defines stress like second order tensors work conjugate to $\nabla \delta u, \nabla \delta a$ and $\nabla \delta b_{i}$ as well as stress like vector quantities work conjugate to $\delta a$ and $\delta b_{i}$. Apart from the equilibrium between the average first Piola-Kirchhoff stresses $P_{m}$ and $P_{f 0}$, the virtual work defined in Eq. (28) expresses equilibria with respect to the first order moment $P_{f 1}$ and the generalized moments $P_{f w(i)}$ and $P_{f g(i)}$.

The only still to be defined component for deploying the virtual work expression of Eq. (28) within a finite-element scheme is an approximation of the integrals in Eqs. (29)-(32), to be provided in the next section. Given closed form expressions or approximations for the aforementioned integrals, the internal virtual work from Eq. (28) is fully defined. A body occupying the domain $\Omega$ in its undeformed state, is in equilibrium under a traction $t$ acting on a portion $\partial \Omega_{N}$ of its boundary $\partial \Omega$ if internal and external virtual works add to zero

$$
\int_{\Omega} \delta W\left(u, a, b_{i}, \xi_{m}, \delta u, \delta a, \delta b_{i}\right) d X+\int_{\partial \Omega_{N}} t \cdot \delta u d X=0 \quad \forall \text { admissible } \delta u, \delta a, \delta b_{i},
$$

which provides sufficient conditions for determining all kinematic fields $u, a$ and $b_{i}$. Eq. (33) implies that the boundary $\partial \Omega$ is assumed to be free of tractions work conjugate to $a$ and $b_{i}$. However, boundary conditions of the Dirichlet kind can still be applied to these variables by restricting the corresponding admissible spaces accordingly.

This article is protected by copyright. All rights reserved. 
An additional equation is necessary regarding the matrix phase plasticity multiplier $\xi_{m}$. For this purpose, Eq. (17) can be enforced by means of a weighted residual discretization of the weak form

$$
\int_{\Omega} \hat{f}\left(\nabla u, a, \xi_{m}\right) \delta \xi_{m} d X=0 \quad \forall \delta \xi_{m}
$$

where $\delta \xi_{m}$ can be considered as a virtual variation of $\xi_{m}$.

\section{DISCRETIZATION}

\subsection{Choice of internal kinematic enrichment functions}

In the derivation of the virtual work expression presented above, the enrichment functions $w_{i}$ and $g_{i}$ were not specified neither in form nor in number. This section presents the set of such functions that are employed in the numerical example to follow. It is straightforward to show that the functions

$$
w_{0}\left(\chi_{f}\right)=\frac{\chi_{f}}{h_{f}} \quad \text { and } \quad w_{1}\left(\chi_{f}\right)=\left(\frac{\chi_{f}}{h_{f}}+\frac{1}{2}\right)^{4}-\frac{1}{5} \quad \text { and } \quad w_{2}\left(\chi_{f}\right)=w_{1}\left(-\chi_{f}\right)
$$

along with

$$
g_{0}\left(\chi_{f}\right)=\frac{h_{f}}{2}\left(\left(\frac{\chi_{f}}{h_{f}}\right)^{2}-\frac{1}{12}\right)
$$

and

$$
g_{1}\left(\chi_{f}\right)=h_{f}\left(\frac{1}{5}\left(\frac{\chi_{f}}{h_{f}}\right)^{5}+\frac{1}{2}\left(\frac{\chi_{f}}{h_{f}}\right)^{4}+\frac{1}{2}\left(\frac{\chi_{f}}{h_{f}}\right)^{3}+\frac{1}{4}\left(\frac{\chi_{f}}{h_{f}}\right)^{2}-\frac{66}{480}\left(\frac{\chi_{f}}{h_{f}}\right)-\frac{13}{480}\right)
$$

and

$$
g_{2}\left(\chi_{f}\right)=g_{1}\left(-\chi_{f}\right)
$$

fulfill the conditions described by Eqs. (7) and (8). Figure 3 visualizes the utilized enrichment functions for $h_{f}=2$, noting that $g_{i}$ functions scale proportional to $h_{f}$ when $w_{i}$ functions are defined as independent of $h_{f}$. The idea behind this specific choice of enrichment functions is that an overall 
variation of the microscopic deformation gradient through the fiber layer should be captured by $w_{0}$, while $w_{1}$ and $w_{2}$ are meant to capture steep variations close to the top and bottom of a fiber layer, respectively. Hence, the performed choice relies on the understanding that for this specific microstructure, damage of a fiber layer under bending will initiate close to its surface. With this in mind, the higher order polynomials in $w_{1}$ and $w_{2}$ can represent steep gradients in the outer quarter of a fiber layer, without affecting significantly the remaining three quarters of the layer.
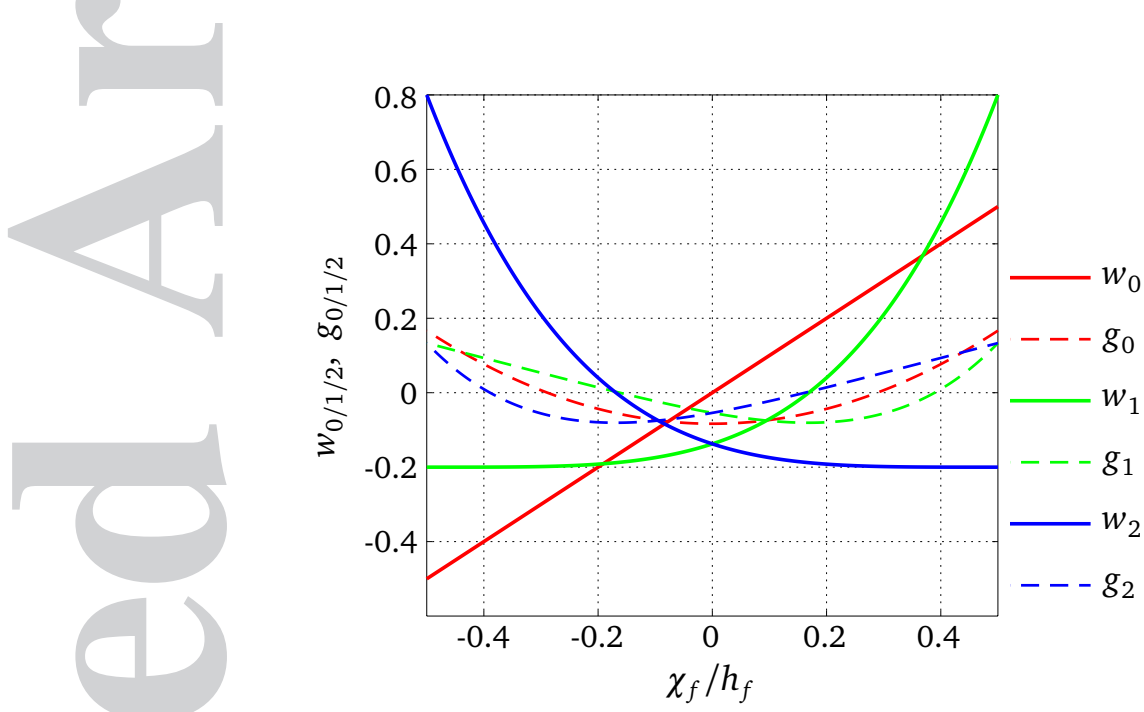

Figure 3. Internal kinematic enrichment functions.

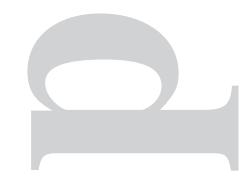

\subsection{Numerical integration over the fiber height}

Different techniques can be employed in order to evaluate the integrals in Eqs. (29)-(32). From standard Gauss quadrature in the unit cell to nonlocal quadrature as proposed in [32]. The present section introduces a special scheme, where numerical integration is performed based on a piecewise linear interpolation of the integrand with four control points A, B, C and D and the shape functions $f_{A}\left(\chi_{f}\right), f_{B}\left(\chi_{f}\right), f_{C}\left(\chi_{f}\right)$ and $f_{D}\left(\chi_{f}\right)$, shown in Figure 4. The locations of the control points at $\chi_{f A}=-h_{f} / 2, \chi_{f B}=-h_{f} / 4, \chi_{f C}=h_{f} / 4$ and $\chi_{f D}=h_{f} / 2$ were chosen for capturing variations close to the surface of a fiber layer, where damage is more likely to initiate, leading to steeper damage gradients.

This article is protected by copyright. All rights reserved. 


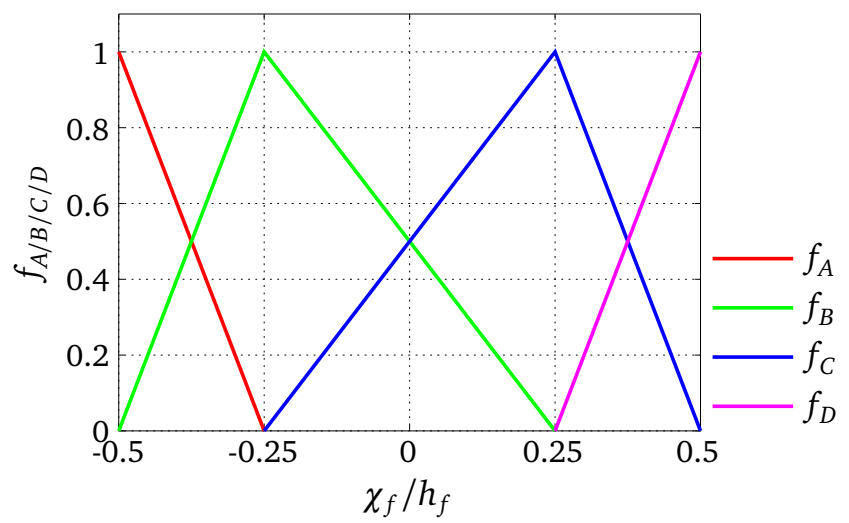

Figure 4. Shape functions for interpolation through the fiber height.

The deformation tensors $F_{f A}, F_{f B}, F_{f C}$ and $F_{f D}$ at the four control points can be evaluated according to Eq. (6) by substituting the corresponding $\chi_{f}$ values as well as the constants

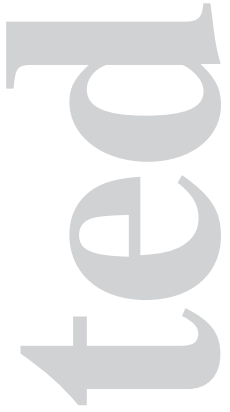

$$
\begin{aligned}
& {\left[w_{0 A}, w_{0 B}, w_{0 C}, w_{0 D}\right]=\left[-\frac{1}{2},-\frac{1}{4}, \frac{1}{4}, \frac{1}{2}\right]} \\
& {\left[w_{1 A}, w_{1 B}, w_{1 C}, w_{1 D}\right]=\left[-\frac{1}{5},-\frac{251}{1280}, \frac{149}{1280}, \frac{4}{5}\right]} \\
& {\left[w_{2 A}, w_{2 B}, w_{2 C}, w_{2 D}\right]=\left[w_{1 D}, w_{1 C}, w_{1 B}, w_{1 A}\right]}
\end{aligned}
$$

and

$$
\begin{aligned}
& {\left[g_{0 A}, g_{0 B}, g_{0 C}, g_{0 D}\right]=\left[\frac{h_{f}}{12},-\frac{h_{f}}{96},-\frac{h_{f}}{96}, \frac{h_{f}}{12}\right]} \\
& {\left[g_{1 A}, g_{1 B}, g_{1 C}, g_{1 D}\right]=\left[\frac{h_{f}}{15}, \frac{259 h_{f}}{15360},-\frac{551 h_{f}}{15360}, \frac{h_{f}}{15}\right]} \\
& {\left[g_{2 A}, g_{2 B}, g_{2 C}, g_{2 D}\right]=\left[g_{1 D}, g_{1 C}, g_{1 B}, g_{1 A}\right]}
\end{aligned}
$$

according to Eqs. (35)-(38).

Assuming also that the corresponding damage values $d_{* A}, d_{* B}, d_{* C}$ and $d_{* D}$ at the previous time instant $t_{*}$ are stored for each of the four control points, the current damage values $d_{A}, d_{B}, d_{C}$ and $d_{D}$ are evaluated based on Eq. (22), as functions of $F_{f A}, F_{f B}, F_{f C}$ and $F_{f D}$ respectively. Finally, the first Piola-Kirchhoff stress tensors $P_{f A}, P_{f B}, P_{f C}$ and $P_{f D}$ at the four control points can be evaluated based on the corresponding deformation tensors through Eqs. (18) and (25), resulting in closed form expressions in terms of $\nabla u, a, \nabla a, b_{0}, \nabla b_{0}, b_{1}, \nabla b_{1}, b_{2}$ and $\nabla b_{2}$. The piecewise linear 
approximation of $\tilde{P}_{f}$ over the fiber height is then given by

$$
\tilde{P}_{f}\left(\chi_{f}\right) \approx P_{f A} f_{A}\left(\chi_{f}\right)+P_{f B} f_{B}\left(\chi_{f}\right)+P_{f C} f_{C}\left(\chi_{f}\right)+P_{f D} f_{D}\left(\chi_{f}\right),
$$

which can be used in order to evaluate the integrals defined in Eqs. (29)-(32), resulting in the approximations

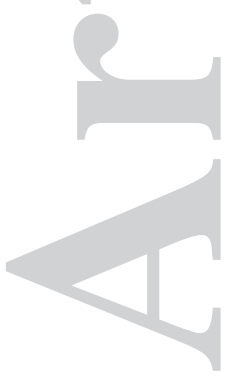

$$
\begin{gathered}
P_{f 0} \approx \frac{1}{8}\left(P_{f A}+3 P_{f B}+3 P_{f C}+P_{f D}\right) \\
P_{f 1} \approx \frac{h_{f}}{96}\left(-5 P_{f A}-6 P_{f B}+6 P_{f C}+5 P_{f D}\right) \\
P_{f w(0)}=\frac{1}{h_{f}} P_{f 1} \\
P_{f w(1)} \approx \frac{1}{30720}\left(-767 P_{f A}-1941 P_{f B}+699 P_{f C}+2009 P_{f D}\right) \\
P_{f w(2)} \approx \frac{1}{30720}\left(2009 P_{f A}+699 P_{f B}-1941 P_{f C}-767 P_{f D}\right) \\
P_{f g(1)} \approx \frac{h_{f}}{860160}\left(5377 P_{f A}+1092 P_{f B}-8400 P_{f C}+1931 P_{f D}\right) \\
P_{f g(2)} \approx \frac{h_{f}}{860160}\left(1931 P_{f A}-8400 P_{f B}+1092 P_{f C}+5377 P_{f D}\right)
\end{gathered}
$$

Eqs. (42)-(49) can be seen as the outcome of a homogenization process, since they provide, in combination with Eq. (26), constitutive relationships between the assumed kinematic variables and their work conjugate stress like quantities in Eq. (28).

\subsection{Choice of finite-element spaces}

Once a set of enrichment functions is chosen for $i \in\{0,1,2\}$, finite-element approximations are required for the unknown vector fields $b_{0}, b_{1}$ and $b_{2}$ along with the ones for $u, \xi_{m}$ and $a$. The numerical results presented in the following section were obtained with meshes consisting of quadrilateral elements, with a nine node quadratic approximation for $u, \xi_{m}$ and $a$ and a four node 
bilinear approximation for $b_{0}, b_{1}$ and $b_{2}$. Other combinations are possible, however, increasing the degree of the interpolation of the $b_{i}$ fields did not result in significant accuracy gain in a number of preliminary calculations. Numerical integration of all terms is performed with nine Gauss points per element.

It should be noted that the ratio of the additional degrees of freedom due to the fields $b_{0}, b_{1}$ and $b_{2}$ can easily be estimated, at least for the interior of the discretized domain. In the interior of the domain, for every four degrees of freedom of a nine node element, there is only one degree of freedom for a four node element. Hence, the ratio of the six components in $b_{0}, b_{1}$ and $b_{2}$ divided by the five components of $u, \xi_{m}$ and $a$ and multiplied by the ratio one to four results in an estimate of $30 \%$ additional degrees of freedom due to the fields $b_{i}$. Hence, the extra computational cost for solving the larger system of equations is rather limited. Of course, there is also some additional cost in the assembly of the right hand side and the stiffness matrix, due to the evaluation of quantities at all four control points A, B, C and D in the unit cell. However, this extra computational load is on one side easy to parallelize and on the other side justified by the considerably more complex material behavior captured by the present model.

(

\subsection{Quasi-static simulation algorithm}

In the context of the composite laminates considered here, mechanical instabilities may arise as global buckling of an overall slender geometry under longitudinal compression or as microbuckling due to compressive stresses along the direction of reinforcement. Additionally, the possibility of a strong material instability due to fiber breakage has to be considered as well. For the simulation of such mechanical instabilities, robust numerical continuation techniques are available. However, the unloading along the unstable equilibrium path performed in such methods may affect the evolution of path dependent phenomena such as plasticity and damage. On the other side, a simulation of the full dynamic problem that occurs across an instability point in reality, is computationally expensive. For the type of materials considered here for instance, the energy released at fiber breakage leads most likely to a highly dynamic response that requires accordingly fine time discretization. Within 
a quasi-static simulation on the other side, it is particularly difficult to solve a load step across an instability and determine a quasi-static equilibrium in the post-buckling or post-damage state. In order to deal with this problem, the present work proposes a quasi-static scheme with a pseudodynamic simulation across instability points.

The main idea is to perform an ordinary quasi-static simulation with a prescribed evolution of loads and boundary displacements at predefined time intervals. A special treatment is performed only if the standard Newton-Raphson algorithm fails to converge to an equilibrium. In that case, a series of rate dependent sub-steps are performed with viscous forces and rate dependent damage evolution until a quasi-static equilibrium is achieved at the end of the prescribed load step. The performed sub-steps do not constitute a real dynamic situation since no inertia is considered and the viscous effects included are artificial ones. Capturing the actual dynamic response during fiber breakage is beyond the scope of the present work and hence a real dynamic simulation that involves a correspondingly high frequency response is avoided. The time interval for each sub-step is heuristically determined aiming at values as large as possible. As the time intervals tend to infinity, a solution that is an equilibrium in a quasi-static sense is recovered. A final sub-step is performed with viscous forces and damage rate dependence completely turned off in order to ensure a quasi-static equilibrium. The flow diagram in Figure 5 defines the considered algorithm more precisely.

The rate independent nonlinear equation for a vanishing $G$ in Figure 5 corresponds to the enforcement of Eqs. (33) and (34), while $G_{\Delta t}$ is a rate dependent version of $G$, discretized in time using a backward Euler approximation for the interval $\Delta t$. Viscous forces with respect to a fixed reference frame are accounted for by including the corresponding virtual work

$$
\delta W_{\mathrm{visc}}=\hat{C}_{\mathrm{visc}} \frac{d \hat{u}}{d t} \cdot \delta \hat{u}=\frac{C_{\mathrm{visc}}}{\Delta t}\left(\left(u-u_{*}\right) \cdot \delta u+\frac{\left(h_{f}+h_{m}\right)^{2}}{12}\left(a-a_{*}\right) \cdot \delta a\right)
$$

to Eq. (33). In Eq. (50), the quantity $\hat{C}_{\text {visc }}$ is a viscosity parameter with units $\mathrm{Ns} \mathrm{mm}^{-4}$ that can vary across the microstructure and $\delta \hat{u}$ is a virtual variation of the non-homogenized displacements field. A piecewise constant parameter $\hat{C}_{\text {visc }}$ with two distinct values $C_{\mathrm{visc}, f}$ and $C_{\mathrm{visc}, m}$ for the fiber 


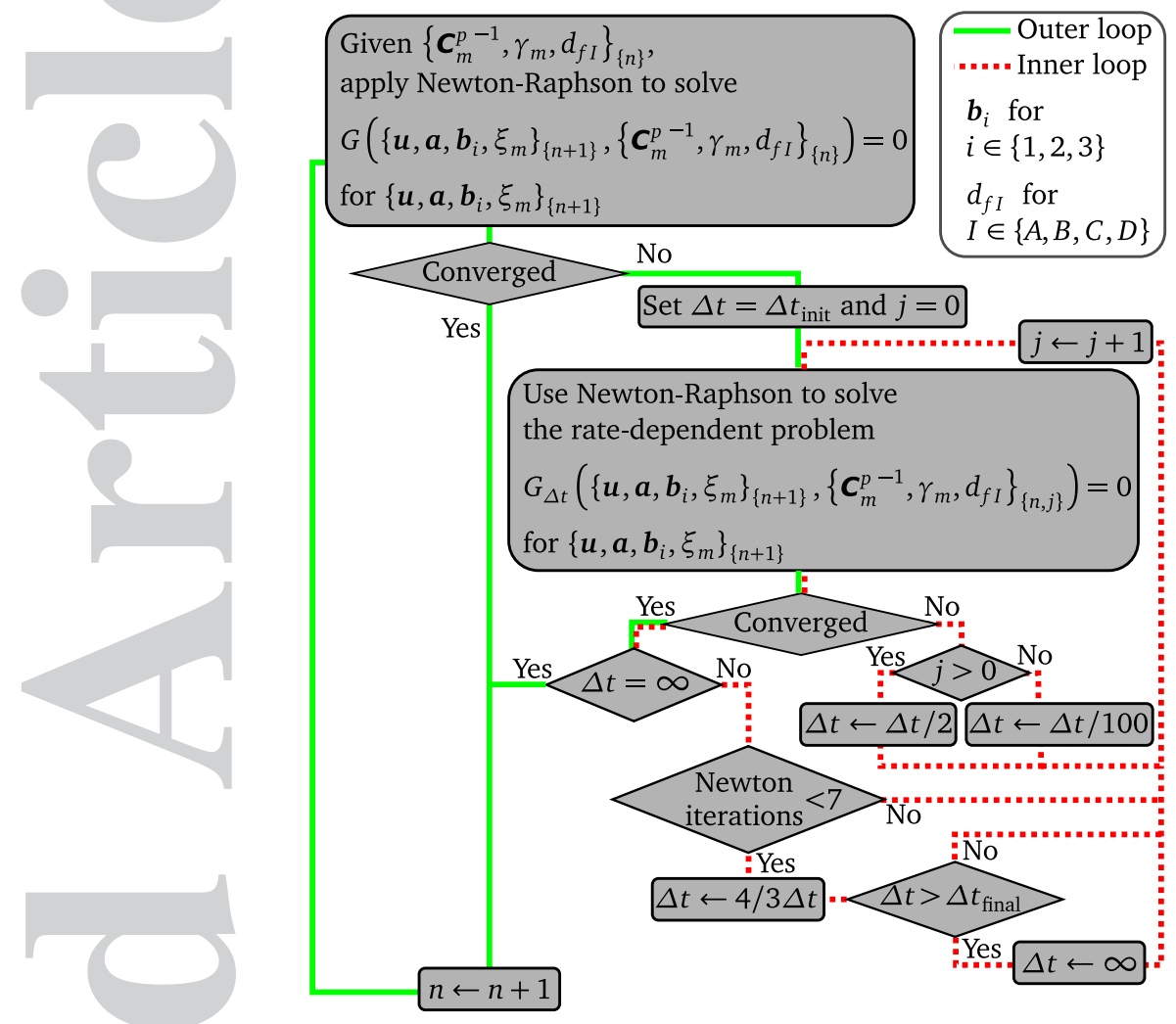

Figure 5. Flow diagram for the quasi-static simulation algorithm.

and matrix, respectively, results in the averaged value $C_{\mathrm{visc}}=c_{f} C_{\mathrm{visc}, f}+c_{m} C_{\mathrm{visc}, m}$ that is employed in the discretized and homogenized approximation included in Eq. (50). Due to the only marginal role of the performed regularization for the quasi-static result at the end of the load step, a detailed derivation of Eq. (50) is omitted here for the sake of brevity. It is however important to note that the asterisk subscript denotes last known solutions for the corresponding fields, which according to Figure 5 correspond to the previous sub-step $j$ posterior to the previous load step $n$.

The rate dependent regularization of the damage evolution in $G_{\Delta t}$ is performed by replacing the closed form Eq. (21) with an ordinary differential equation with respect to time, defined as

$$
\dot{\tilde{d}}=\frac{\max \left\{\tilde{d}_{\text {trial }}, \tilde{d}_{*}\right\}-\tilde{d}}{C_{d, \text { visc }}} .
$$

This article is protected by copyright. All rights reserved. 
At steady state, the rate $\dot{\tilde{d}}$ tends to zero and Eq. (51) recovers Eq. (22). A backward Euler discretization of Eq. (51) yields

$$
\tilde{d}=\frac{C_{d, \text { visc }} \tilde{d}_{*}+\Delta t \max \left\{\tilde{d}_{\text {trial }}, \tilde{d}_{*}\right\}}{C_{d, \text { visc }}+\Delta t},
$$

which is finally used in the definition of $G_{\Delta t}$ instead of Eq. (22) used in the rate independent problem.

The rate dependent models introduced in this section are not essential for the quasi-static equilibrium obtained at the end of a load step across an instability. However, the ratio between the damage viscosity $C_{d \text {,visc }}$ and the artificial damping parameter $C_{\text {visc }}$ determines whether damage evolution is fast or slow compared to the dynamics of the system in terms of displacements. Depending on this ratio, different damage propagation paths are in principle possible, nevertheless this effect is not investigated in the present work.

\section{NUMERICAL RESULTS}

The numerical results presented in this section refer to a reinforced beam with an initial geometrical imperfection subjected to longitudinal compression. The example considered involves global buckling leading to large deformations and a combination of compressive and bending stresses within individual fiber layers. Due to the gradient of stresses through the fiber thickness, damage evolves non uniformly, starting from the outer edges. At the same time large shear strains arising in the matrix layers are accommodated by plastic deformation. The combination of the aforementioned nonlinear effects at the macro and micro scales make this example suitable for the evaluation of the model proposed above.

Figure 6 shows the geometry and boundary conditions of the example considered here along with the discretization corresponding to a non-homogenized model that provides a direct simulation based reference solution. The illustrated beam of length $L=4 \mathrm{~mm}$ and height $H=1 \mathrm{~mm}$ contains 


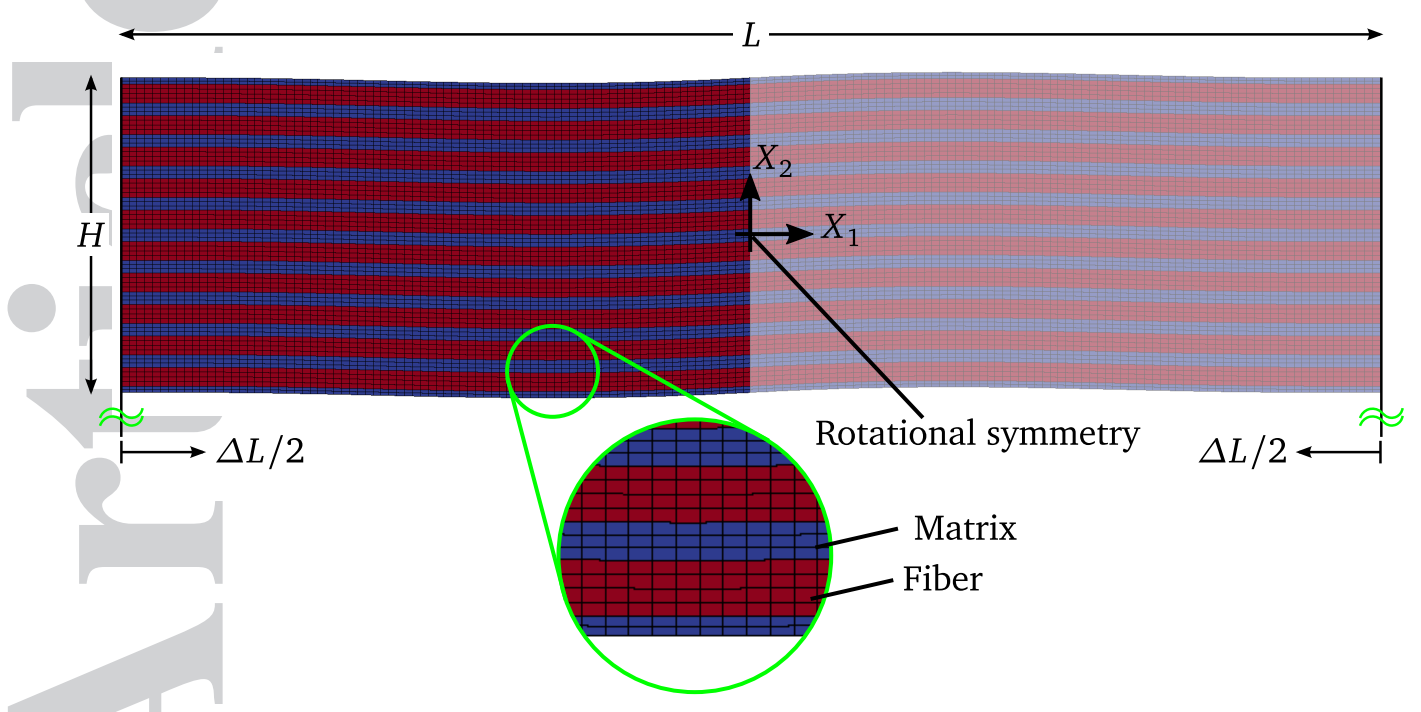

Figure 6. Reference model for direct simulation.

10 reinforcing fiber layers occupying a fraction $c_{f}=0.6$ of the total volume. The Young's and shear moduli of the fiber material are $E_{f}=2.510^{5} \mathrm{MPa}$ and $G_{f}=10^{5} \mathrm{MPa}$ respectively, while the corresponding values for the matrix phase are $E_{m}=1250 \mathrm{MPa}$ and $G_{m}=500 \mathrm{MPa}$. The numerical results of this section include the case of a fully elastic matrix phase as well as the case of an elastoplastic matrix material with initial yield stress $\sigma_{y m}=2.5 \mathrm{MPa}$ and linear isotropic hardening modulus $H_{m}=62.5 \mathrm{MPa}$. With respect to the quasi-brittle damage behavior of the fiber phase, different values of the damage initiation threshold $r_{\text {th }}$ are considered between 2 and $6 \sqrt{\mathrm{N}} / \mathrm{mm}$ as well as two alternative values of the damage evolution slope $C_{d}$ equal to 0.4 and $0.8 \mathrm{~mm} / \sqrt{\mathrm{N}}$. For this set of damage parameters, Figure 7 shows the stress versus strain response of a single fiber under uniaxial tension. The residual integrity parameter $\omega_{\infty}$ is set equal to $5 \cdot 10^{-4}$ in all cases.

The left and right hand sides of the beam in Figure 6 are interlinked by a periodicity condition superimposed with a longitudinal shortening $\Delta L$. Both conditions can be expressed as

$$
\left.u\right|_{X_{1}=-L / 2}=\left.u\right|_{X_{1}=L / 2}+\left(\begin{array}{c}
\Delta L \\
0
\end{array}\right)
$$

This article is protected by copyright. All rights reserved. 


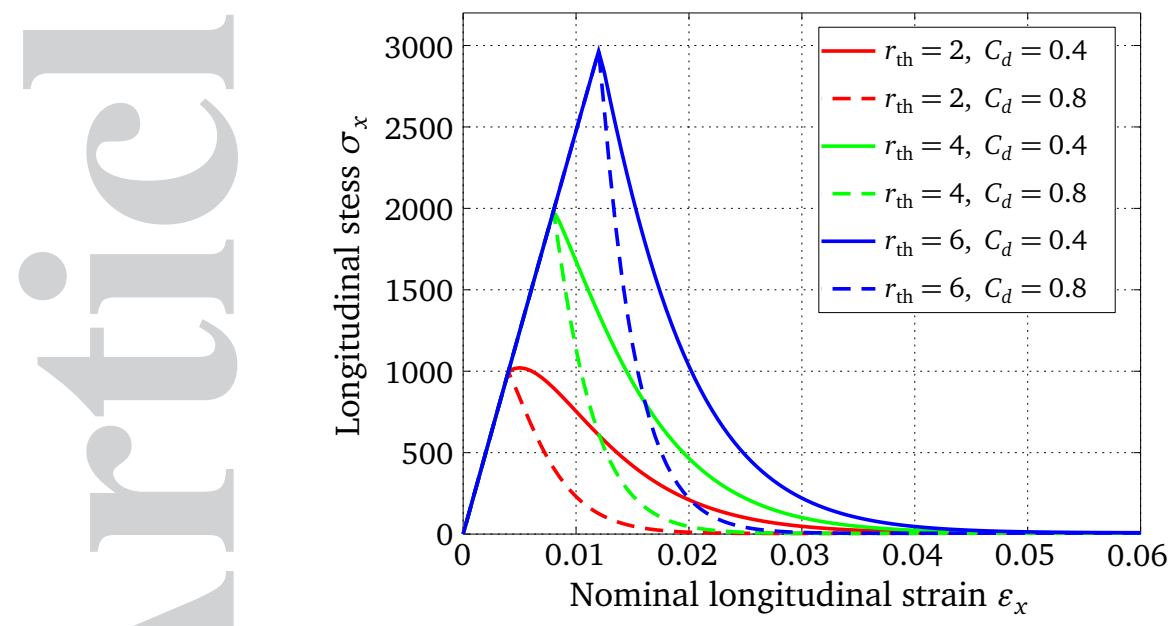

Figure 7. Uniaxial tension response of a single fiber for different damage parameters $r_{\text {th }}$ and $C_{d}$.

At the same time, the beam geometry includes a geometrical imperfection in form of a waviness defined through the expression

$\Delta X_{2}\left(X_{1}\right)= \begin{cases}\frac{a_{w}}{2} \sin \left(\frac{2 \pi X_{1}}{L_{w}}\right)\left(1-\left|\frac{2 \pi X_{1}}{L_{w}}\right|\right) & \text { for }\left|X_{1}\right| \leq L_{w} / 2 \\ 0 & \text { elsewise, }\end{cases}$

where $a_{w}=0.06 \mathrm{~mm}$ and $L_{w}=3.6 \mathrm{~mm}$ are amplitude and wavelength parameters respectively.

Due to the available symmetries in the boundary conditions and geometry, it is sufficient to model only the non faded half of the domain illustrated in Figure 6 with a twofold rotational symmetry constraint along the vertical centerline. Of course, the enforcement of the aforementioned symmetries limits the available global buckling modes to those with a period along the horizontal direction equal to $L$ and its fractions.

Figures 8 and 9 summarize all performed simulations in terms of the obtained compressive forces as a function of the applied compressive strain for a purely elastic and an elastoplastic matrix material respectively. All graphs in the left column correspond to the lower value of $C_{d}$ which represents a comparatively less brittle fiber phase, while the graphs on the right hand side correspond to the higher value of $C_{d}$ and a more brittle variant of the reinforcing fibers.

This article is protected by copyright. All rights reserved. 

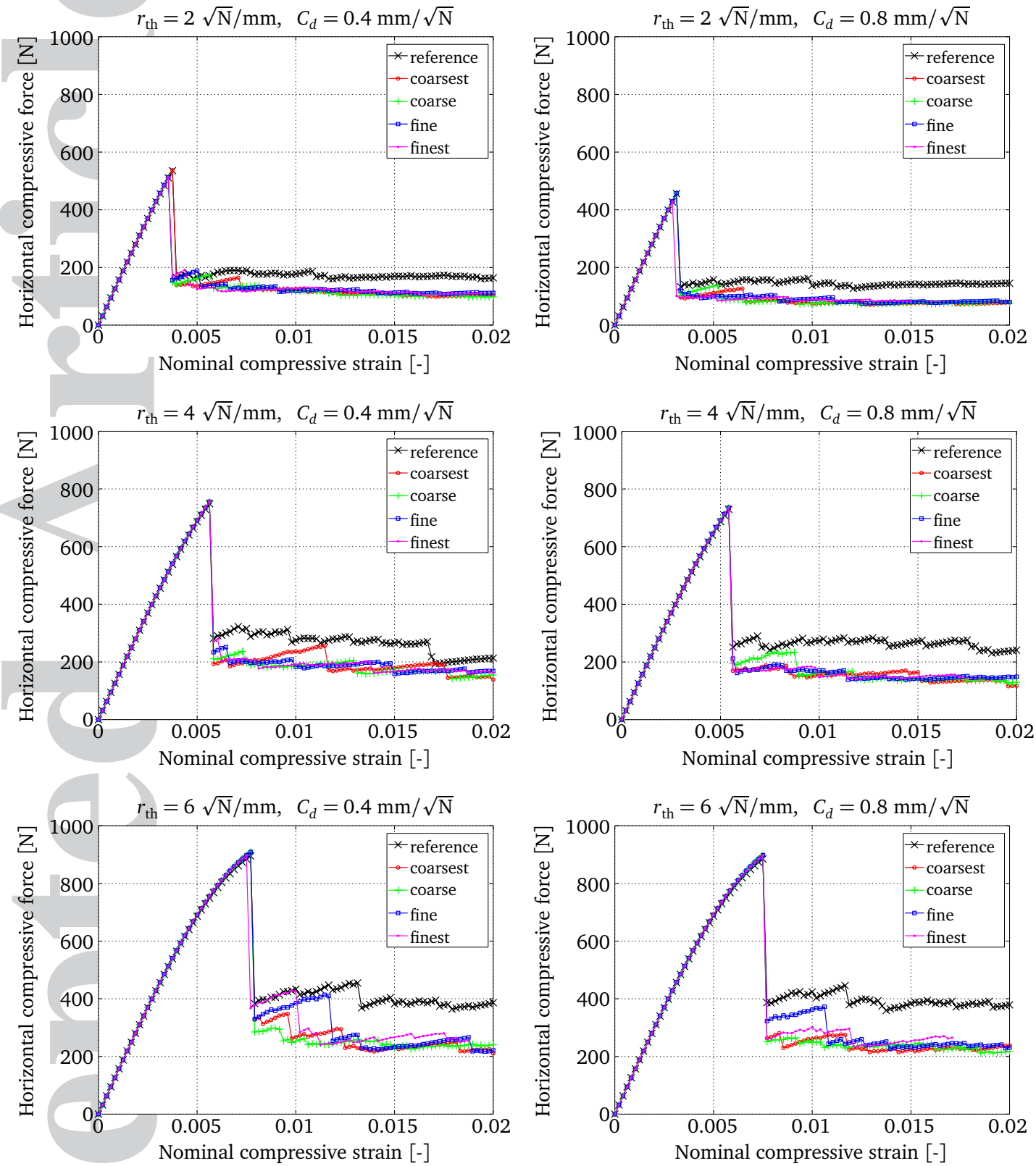

Figure 8. Compressive load versus compressive strain for elastic matrix and different values of the damage threshold parameter $r_{\mathrm{th}}$ (row wise) and the damage slope parameter $C_{d}$ (column wise).

Each diagram presents results obtained with the reference model of Figure 6 as well as results obtained with the proposed homogenized model for four different meshes. From the coarsest to the finest, the four meshes consist of 16 by 16,20 by 20,24 by 24 and 28 by 28 elements respectively, used to discretize the non faded half of the geometry shown in Figure 6 with dimensions $L / 2$ by $H$. The corresponding element sizes are $0.0625,0.05,0.0417$ and $0.0357 \mathrm{~mm}$ in the vertical direction This article is protected by copyright. All rights reserved. 

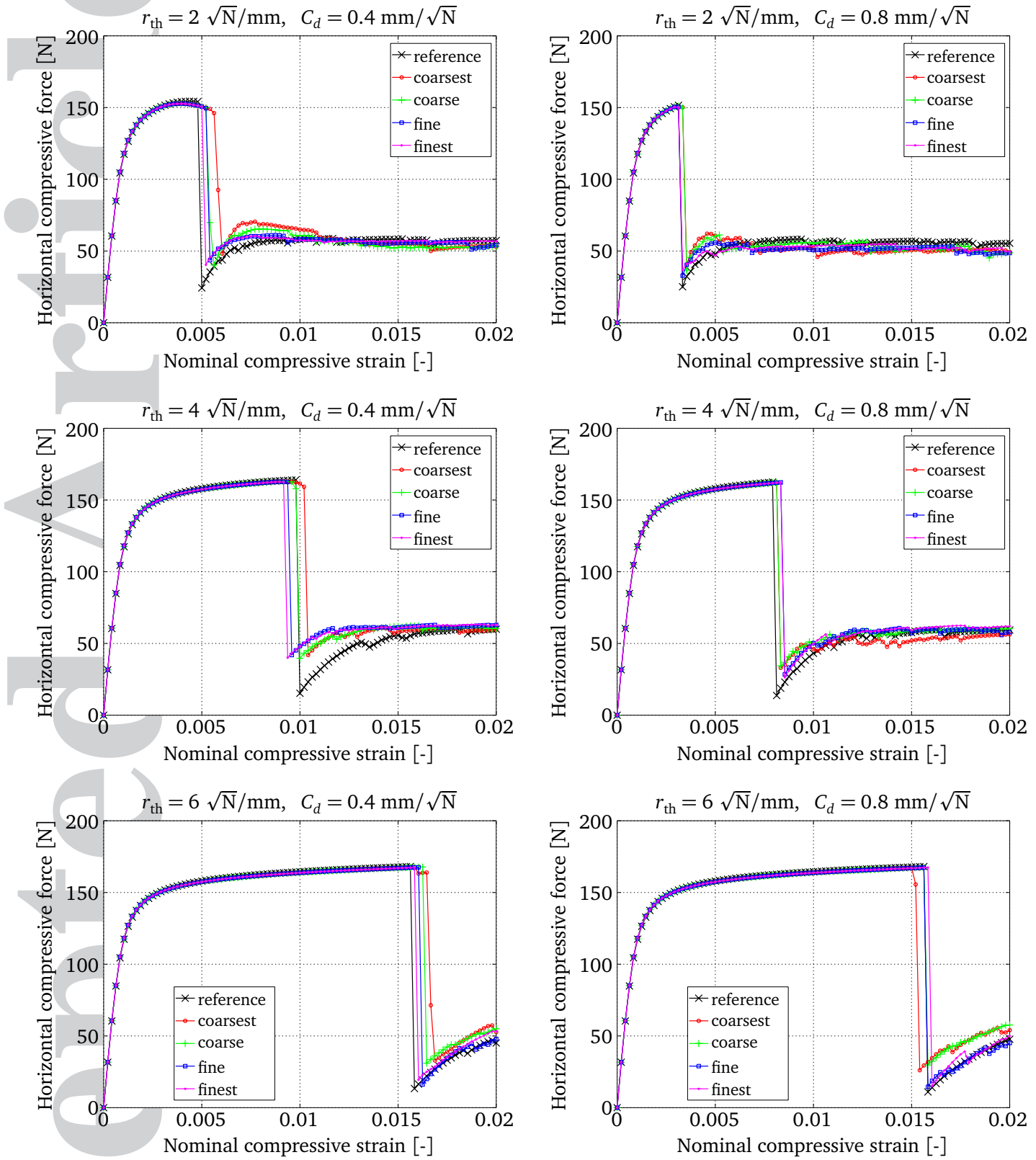

Figure 9. Compressive load versus compressive strain for elastoplastic matrix and different values of the damage threshold parameter $r_{\mathrm{th}}$ (row wise) and the damage slope parameter $C_{d}$ (column wise).

and double as much in the horizontal direction. Based on the data provided in the beginning of this section, the fiber height is equal to $0.06 \mathrm{~mm}$ and therefore comparable to the mesh sizes for the homogenized model. For comparison, a fiber layer in the the reference model is discretized through four elements of $0.015 \mathrm{~mm}$.

This article is protected by copyright. All rights reserved. 
The obtained curves are obtained by incrementing the prescribed nominal compressive strain from zero to 0.02 in 96 equisized intervals. In general, the results from the homogenized model seem to capture the force response curves up to the instability as well as the instability point remarkably well for all employed meshes. For a purely elastic matrix material the instability is captured with no more than one load step discrepancy in all cases. The predicted instability points for an elastoplastic material deviate by four load steps in the worst case. The accuracy of the homogenized model with respect to the reference model is in general better for the higher value of the damage slope parameter $C_{d}$ and hence for comparatively more brittle fibers. The post-instability response is also reproduced fairly accurately, especially in the case of an elastoplastic matrix phase.

Compared to Figure 8, the weakening within the compressive strain range from 0.001 to 0.003 shown in Figure 9 is due to matrix plasticity induced buckling and is not related to fiber damage. For high values of the damage threshold parameter $r_{\mathrm{th}}$, fiber damage initiates at considerably high compressive strain values, far in the post-buckling regime.

Figure 10 contains an example of a comparison between a local field and the corresponding homogenized fields. It actually shows the equivalent strain energy $\tilde{r}$ through a vertical cross section of the considered structure as calculated by the reference model as well as the corresponding homogenized fields $r_{A}$ and $r_{D}$ for the fiber bottom and the top respectively, according to subsection 4.2. The provided snapshot corresponds to the load step just before the collapse point, while the considered cross section traverses the site of the imminent fiber breakage. The circular marks identify points of the reference solution that are approximated by the continuous curves of the homogenized solution. Apart from the regions close to the bottom and top edges of the beam, where discrepancies appear due to edge effects, there is decent agreement between the local and the homogenized fields.

Figures 11 and 12 depict damage fields at instants immediately after the corresponding collapse points for a purely elastic and an elastoplastic matrix phase respectively. The illustrated homogenized fields correspond to the damage variable $d_{D}$ at the top of the fiber, while the corresponding fields at control points $\mathrm{A}, \mathrm{B}$ and $\mathrm{C}$ are not included here although they are also 


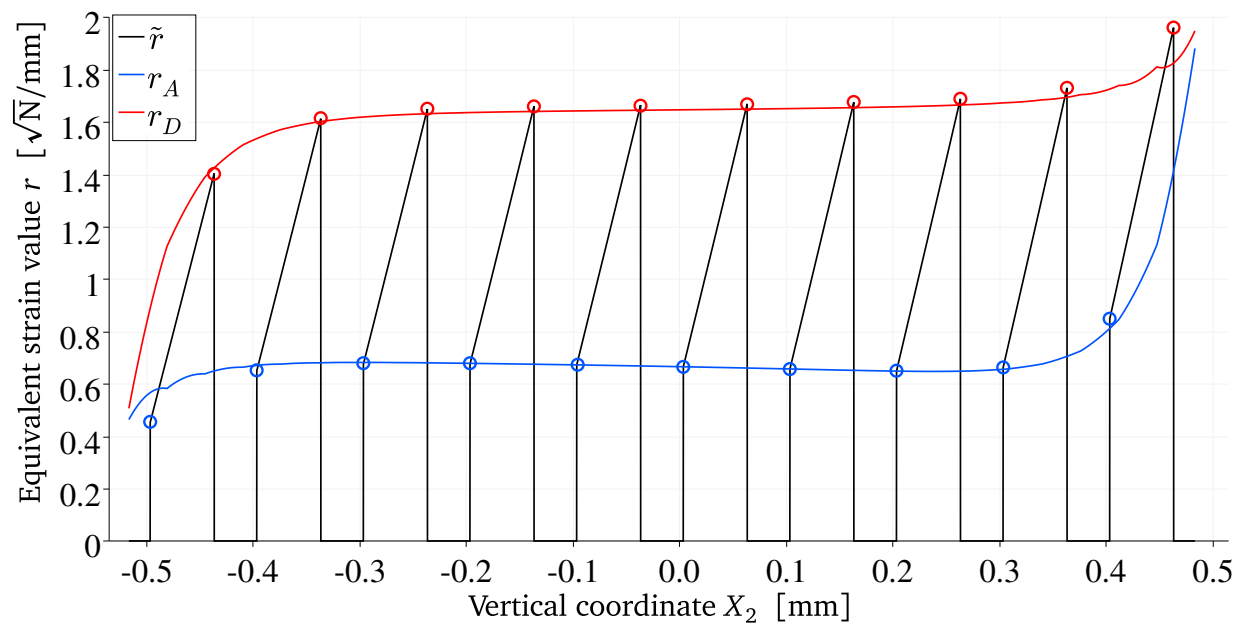

Figure 10. Comparison between the equivalent strain values from the reference model and the homogenized model (mesh $28 \times 28$ ) for elastic matrix material, damage threshold $r_{t h}=2 \sqrt{N} / \mathrm{mm}$, damage slope parameter $C_{d}=0.8 \mathrm{~mm} / \sqrt{\mathrm{N}}$, through the cross section at $X_{1}=-0.56 \mathrm{~mm}$ and at load step 14 (nominal compressive strain 0.00291).

available. The two figures demonstrate that the proposed homogenized model captures both the location of fiber breakage and its extent rather accurately. The color range covers the damage interval from zero (blue) to one (red).

The numerical study presented in this section showcases the capability of the proposed homogenization method to capture the underlying micromechanical behavior in a rather broad range of configurations involving finite deformations, plasticity and quasi-brittle damage effects. Without resorting to discontinuous methods, cases of damage localization as in the provided examples require relatively fine meshes and therefore they limit the gain in terms of degrees of freedom between the reference and the homogenized models in the order of a factor of five. Nevertheless, the versatility of the homogenized model due to the decoupling between the computational mesh and the material microstructure, allows for significant optimizations such as adaptive mesh refinement and selective activation of the internal kinematic variables $b_{i}$. In combination with such techniques, the potential computational gain of the proposed method compared to direct simulations is considerable.

Among other multiscale approaches, reviewed in the introduction, the characteristics of the present example limit the possible choices for achieving comparable results, rather drastically. In order to account for fiber bending effects, a second order homogenization scheme would be 
necessary. In combination with the strongly nonlinear material behavior and kinematics, this leads to second order $\mathrm{FE}^{2}$ as the only obvious choice. $\mathrm{An} \mathrm{FE}^{2}$ model of this level of complexity was developed in [33] and was used for solving a corresponding two dimensional problem, where only a marginal computational gain compared to a direct model was reported.
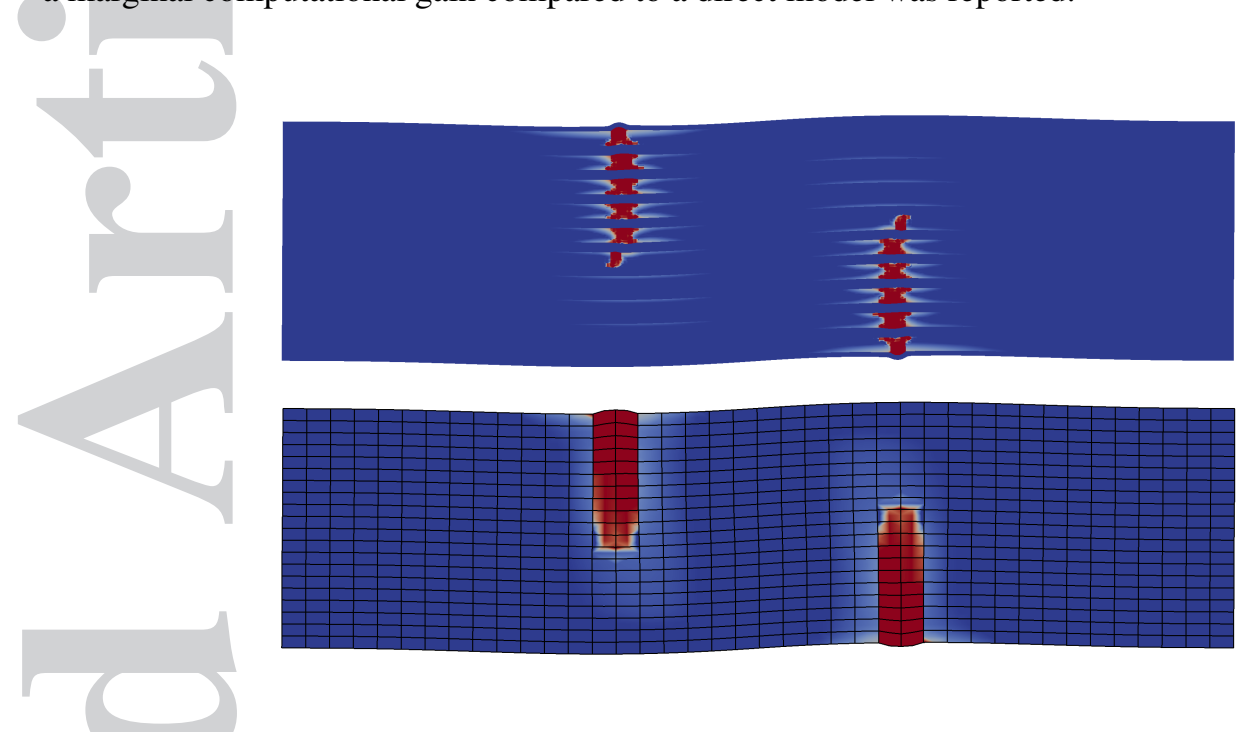

Figure 11. Damage variable $\tilde{d}$ for the reference model (top) and damage variable $d_{D}$ at the top of the fiber for the homogenized model with a $20 \times 20$ mesh (bottom), for elastic matrix material, damage threshold $r_{t h}=2 \sqrt{N} / \mathrm{mm}$, damage slope parameter $C_{d}=0.4 \mathrm{~mm} / \sqrt{N}$, at the first load step after the instability point (load step 19, nominal compressive strain 0.004).

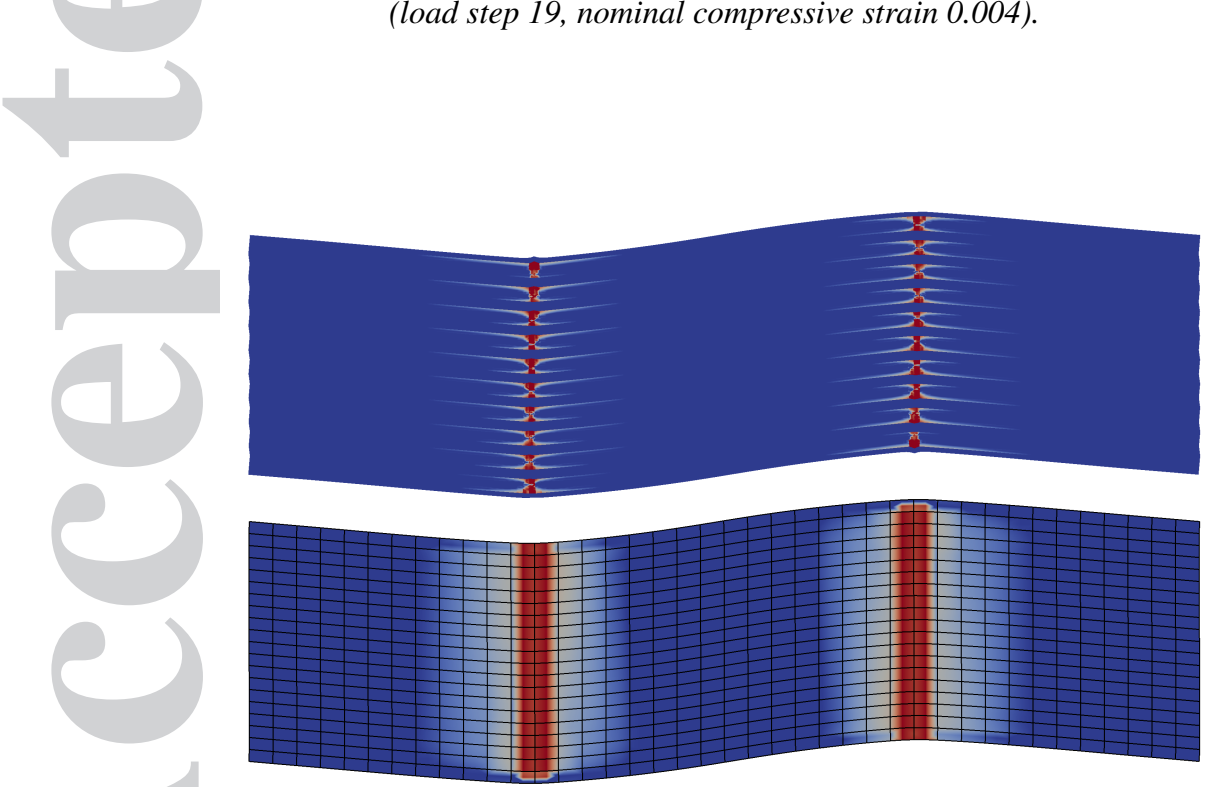

Figure 12. Damage variable $\tilde{d}$ for the reference model (top) and damage variable $d_{D}$ at the top of the fiber for the homogenized model with a $20 \times 20$ mesh (bottom), for elastoplastic matrix material, damage threshold $r_{\text {th }}=2 \sqrt{\mathrm{N}} / \mathrm{mm}$, damage slope parameter $C_{d}=0.4 \mathrm{~mm} / \sqrt{\mathrm{N}}$, at the first load step after the instability point (load step 27, nominal compressive strain 0.0056). 


\section{CONCLUDING REMARKS}

The present work demonstrates how a rich kinematic description at the macroscale can yield a high fidelity representation of a periodic microstructure in the presence of complex inelastic behavior at the microscale under large deformations and strains. Given a specific choice of internal kinematic variables and constitutive laws at the microscale, a coupled system of equations for the homogenized displacements and the internal kinematic variables is formulated at the macroscale through an approximate integration over the unit cell. As an outcome of this process, stress like quantities work conjugate to the corresponding kinematic variables are obtained in closed form. Although these expressions can be seen as homogenized constitutive laws describing stress like quantities in terms of all kinematic variables, it should be highlighted here that these intermediate abstraction of stresses is only a technicality, not essential for the formulation. Essential for the proposed formulation are (i) the definition and choice of internal kinematic variables, (ii) the microscale constitutive laws and (iii) an integration method for the virtual work over the unit cell. With these three components given, the remaining steps towards the final finite-element model defined at the macroscale arise as natural consequences.

Compared to previous implementations of the aforementioned methodology, the higher complexity of microstructural phenomena covered by present work requires a richer kinematic description resulting in an increased number of degrees of freedom. At the same time, quasi-brittle behavior of the fiber material is modeled by means of a strain based damage model which avoids the need for further degrees of freedom related to damage and relies only on corresponding state variables stored at the last known time instant. With respect to an integration over the unit cell, the present work provides an approximate integration scheme based on four control points through the fiber thickness in addition to a single point within the matrix layer.

The excellent reproduction of the direct simulation results by the homogenized model in the numerical example presented justifies the additional computational cost due to the internal kinematic variables adopted. Global collapse of the simulated structure is predicted accurately both in terms 
of applied load and nominal strain. At the same time, comparisons of local quantities from the reference non-homogenized model with corresponding values obtained by the homogenized model are indicative for the high fidelity level of the method.

The proposed methodology is in general applicable to a broader range of constitutive laws compared to the isotropic ductile and brittle materials considered here. Corresponding orthotropic constitutive laws, with different material properties parallel and perpendicular to the layering direction are rather straightforward to implement. For material combinations with a lower stiffness contrast between the two phases, or for a combination of two ductile phases, a richer description of ductile layers is probably required. In such cases, variations through the ductile layer thickness could be captured by using kinematic enhancements and integration schemes similar to the ones suggested here for the brittle phase. Extensions of the proposed method to different types of damage, such as matrix cracking and interface debonding are also conceptually possible. Moreover, an extension to three dimensional layered structures is very straightforward while a possible extension to unidirectionally reinforced composites requires some more advanced steps. The unit cell with a circular inclusion in that case would require additional internal kinematic variables, an appropriate choice of control points within the fiber and the matrix materials and the definition of a corresponding numerical integration scheme. However, the margin for a possible computational gain is also larger due to the higher computational complexity of the direct modeling approach in three dimensions. A comparison of the numerical performance of the aforementioned three dimensional extensions of the present work with other high fidelity approaches such as multilevel finite-element methods would be interesting.

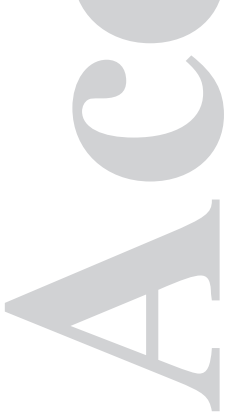

\section{ACKNOWLEDGEMENTS}

This work was supported by the Danish Council for Independent Research, through the Sapere Aude project "Higher Order Theories in Solid Mechanics".

This article is protected by copyright. All rights reserved. 


\section{REFERENCES}

1. Fleck NA. Compressive failure of fiber composites. Advances in Applied Mechanics 1997; 33:43-117.

2. Dalgleish BJ, Trumble KP, Evans AG. The strength and fracture of alumina bonded with aluminum-alloys. Acta Metallurgica 1989; 37(7):1923-1931.

3. Schrenk WJ, Alfrey T. Some physical properties of multilayered films. Polymer Engineering and Science 1969; 9(6):393-399.

4. Kyriakides S, Arseculeratne R, Perry EJ, Liechti KM. On the compressive failure of fiber-reinforced composites. International Journal of Solids and Structures 1995; 32(6-7):689-738.

5. Jha M, Charalambides P. A finite element analysis of fracture initiation in ductile/brittle periodically layered composites. International Journal of Fracture 1998; 90(4):299-323.

6. Orifici AC, Herszberg I, Thomson RS. Review of methodologies for composite material modelling incorporating failure. Composite Structures 2008; 86(1-3):194-210.

7. Voyiadjis G, Deliktas B. A coupled anisotropic damage model for the inelastic response of composite materials. Computer Methods in Applied Mechanics and Engineering 2000; 183(3-4):159-199.

8. Chaboche J, Maire J. A new micromechanics based CDM model and its application to CMC's. Aerospace Science and Technology 2002; 6(2):131-145.

9. Maimi P, Camanho PP, Mayugo JA, Davila CG. A continuum damage model for composite laminates: Part I constitutive model. Mechanics of Materials 2007; 39(10):897-908.

10. Basu S, Waas AM, Ambur DR. Prediction of progressive failure in multidirectional composite laminated panels. International Journal of Solids and Structures 2007; 44(9):2648-2676.

11. Kanoute P, Boso DP, Chaboche JL, Schrefler BA. Multiscale methods for composites: A review. Archives of Computational Methods in Engineering 2009; 16(1):31-75.

12. Geers M, Kouznetsova V, Brekelmans W. Multi-scale computational homogenization: Trends and challenges. Journal of Computational and Applied Mathematics 2010; 234(7):2175 - 2182.

13. Ortolano J, Hernández Ortega JA, Oliver Olivella X. A comparative study on homogenization strategies for multiscale analysis of materials. Centre Internacional de Mètodes Numèrics en Enginyeria (CIMNE), 2013.

14. Blanco PJ, Sanchez PJ, de Souza Neto EA, Feijoo RA. Variational foundations and generalized unified theory of RVE-based multiscale models. Archives of Computational Methods in Engineering 2016; 23(2):191-253.

15. Neron D, Ladeveze P. Proper generalized decomposition for multiscale and multiphysics problems. Archives of Computational Methods in Engineering 2010; 17(4):351-372.

16. Fritzen F, Leuschner M. Nonlinear reduced order homogenization of materials including cohesive interfaces. Computational Mechanics 2015; 56(1):131-151.

17. Mesarovic SD, Padbidri J. Minimal kinematic boundary conditions for simulations of disordered microstructures. Philosophical Magazine 2005; 85(1):65-78.

This article is protected by copyright. All rights reserved. 
18. Saeb S, Steinmann P, Javili A. Aspects of computational homogenization at finite deformations: A unifying review from Reuss' to Voigt's bound. Applied Mechanics Reviews 2016; 68(5):050 801.

19. Forest S. Micromorphic Media. Springer: Vienna, 2013; 249-300.

20. Forest S. Mechanics of generalized continua: construction by homogenizaton. Le Journal De Physique IV 1998; 08(PR4):39-48.

21. De Bellis ML, Addessi D. A Cosserat based multi-scale model for masonry structures. International Journal for Multiscale Computational Engineering 2011; 9(5):543-563.

22. Addessi D. A 2D Cosserat finite element based on a damage-plastic model for brittle materials. Computers and Structures 2014; 135:20-31.

23. Fleck NA, Shu JY. Microbuckle initiation in fibre composites: a finite element study. Journal of the Mechanics and Physics of Solids 1995; 43(12):1887-1918.

24. Poulios K, Niordson CF. Homogenization of long fiber reinforced composites including fiber bending effects. Journal of the Mechanics and Physics of Solids 2016; 94:433-452.

25. Oller S, Oñate E, Miquel J, Botello S. A plastic damage constitutive model for composite materials. International Journal of Solids and Structures 1996; 33(17):2501-2518.

26. Fish J, Yu Q, Shek K. Computational damage mechanics for composite materials based on mathematical homogenization. International Journal for Numerical Methods in Engineering 1999; 45(11):1657-1679.

27. Chaboche J, Kruch S, Maire J, Pottier T. Towards a micromechanics based inelastic and damage modeling of composites. International Journal of Plasticity 2001; 17(4):411-439.

28. Haj-Ali R, Aboudi J. Formulation of the high-fidelity generalized method of cells with arbitrary cell geometry for refined micromechanics and damage in composites. International Journal of Solids and Structures 2010; 47(2526):3447-3461.

29. Neff P, Ghiba ID, Lankeit J. The exponentiated Hencky-logarithmic strain energy. Part I: Constitutive issues and rank-one convexity. Journal of Elasticity 2015; 121(2):143-234.

30. Eterovic A, Bathe KJ. A hyperelastic-based large strain elastoplastic constitutive formulation with combined isotropic-kinematic hardening using the logarithmic stress and strain measures. International Journal for Numerical Methods in Engineering 1990; 30(6):1099-1114.

31. Simo JC, Ju JW. Strain- and stress-based continuum damage models. I. formulation. International Journal of Solids and Structures 1987; 23(7):821-840.

32. Fish J, Kuznetsov S. Computational continua. International Journal for Numerical Methods in Engineering 2010; 84(7):774-802.

33. Nguyen VD, Noels L. Computational homogenization of cellular materials. International Journal of Solids and Structures 2014; 51(11):2183 - 2203.

This article is protected by copyright. All rights reserved. 